\title{
Potência irradiada por uma carga elétrica acelerada no espaço-tempo de Minkowski
}

Power radiated by an accelerated electric charge in Minkowski spacetime

\author{
Damião Pedro Meira Filho*1@, Jorge Kysnney Santos Kamassury \\ ${ }^{1}$ Instituto Federal do Pará, Santarém, PA, Brasil \\ ${ }^{2}$ Universidade Federal do Oeste do Pará, Instituto de Engenharia e Geociências, Santarém, PA, Brasil
}

Recebido em 20 de Dezembro, 2017. Revisado em 30 de Janeiro, 2018. Aceito em 12 de Fevereiro, 2018.

\begin{abstract}
No presente artigo empregaremos a Teoria de Campos Clássicos para abordar o campo eletromagnético e a interação de uma carga elétrica acelerada com esse campo no espaço-tempo de Minkowski. Para tanto, apresentamos primeiramente as propriedades matemáticas do espaço-tempo em questão e as etapas para a obtenção das equações do campo eletromagnético com fonte a partir de uma formulação lagrangeana desse campo. Em seguida, utilizamos o formalismo das funções de Green para encontrar as soluções das equações de campo, desenvolvendo inclusive os passos para a obtenção do propagador de Feynman. Por fim, alcançamos os potenciais de Liénard-Wiechert e as expressões dos campos de radiação elétrico e magnético, apresentando posteriormente o padrão de radiação emitida por uma carga em condição não-relativística, a fórmula da potência de Larmor e sua forma covariante assim como a generalização de Liénard.
\end{abstract}

Palavras-chave: Potência de Larmor, Generalização de Liénard, Teoria de campos clássicos, Espaço-tempo de Minkowski, Cálculo de radiação.

In this paper we will use the Classical Fields Theory to address the electromagnetic field and the interaction of an accelerated electric charge with this field in Minkowski spacetime. For this, we first present the mathematical properties of the spacetime in question and the steps to obtain the electromagnetic field equations with source from a lagrangean formulation of that field. Then, we use Green's formalism to find the solutions of the field equations, including the steps to obtain Feynman propagator. Finally, we reach the potential of Liénard-Wiechert and the expressions of the fields of electric and magnetic radiation, presenting later the radiation pattern emitted by a charge in non-relativistic condition, the Larmor formula and its covariant form besides the Liénard's generalization. Keywords: Larmor formula, Liénard's generalization, Theory of classical fields, Minkowski spacetime, Calculation of radiation.

\section{Introdução}

Os estudos sobre o fenômeno da emissão de radiação vêm sendo realizados desde meados do século XIX por vários físicos teóricos e experimentais. No início, esses estudos visavam investigar e explicar a radiação térmica emitida por corpos incandescentes. Ainda que muitas explicações fossem apresentadas, poucas eram coerentes com o referido fenômeno.

Na segunda metade do século XIX, a radiação térmica e outros efeitos recém observados, como o raio-X, passaram a ser analisados com base na teoria eletromagnética que, por sua vez, ganhava consistência matemática nos trabalhos do físico e matemático escocês James Clerk Maxwell (1831-1879).

Influenciado pelos trabalhos do físico e químico inglês Michael Faraday (1791-1867) e do engenheiro, físico e matemático irlandês Sir William Thomson (1824-1907), Maxwell apresentou suas contribuições ao eletromagne-

*Endereço de correspondência: damiao.meira@ifpa.edu.br tismo principalmente em três artigos [1], a saber: On Faraday's Lines of Force (1856) [2], On Physical Lines of Force (1861) [3] e A Dynamical Theory of the Electromagnetic Field (1864) [4]. Todavia, é na sua célebre obra $A$ treatise on Eletricity and Magnetism publicada em 1873 [5], que Maxwell apresenta uma síntese coerente do magnetismo, da óptica e da eletricidade (com resultados dos artigos anteriores), unificando tais domínios [6]. Convém mencionar que a Teoria Eletromagnética foi a primeira a ser desenvolvida efetivamente com base no conceito de campo, nesse caso, campo clássico.

Ainda nesse período, importantes trabalhos foram desenvolvidos objetivando, por exemplo, a solução da equação de onda não-homogênea. Em 1858, o matemático alemão Bernhard Riemann (1826-1866) considera os potencias escalar e vetorial em instantes retardados para solucionar tal equação. O uso pioneiro do conceito de potenciais retardados por Riemann, entretanto, só veio a ser divulgado através do seu trabalho intitulado $A$ Contribution to Electrodynamics [7] em 1867, mesmo ano 
em que o físico dinamarquês Ludwig Lorenz (1829-1891), publicou o trabalho On the Identity of the Vibration of Light with Electrical Currents [8], onde este sugere o uso dos potenciais retardados e emprega o chamado gauge (calibre) de Lorenz que inclusive o mesmo já havia utilizado em um trabalho publicado em 1861 ao estudar ondas de elasticidade 9 .

Aplicações dessas soluções a problemas específicos foram apresentadas independentemente pelo físico e engenheiro francês Alfred-Marie Liénard (1869-1958), em 1898, e pelo geofísico alemão Emil Wiechert (1861-1928) em 1900 10. Estas soluções são conhecidas hoje como potenciais (escalar e vetorial) retardados de LiénardWiechert.

A importante demonstração de que uma carga elétrica acelerada emitia ondas eletromagnéticas foi realizada pelo físico e matemático inglês Sir Joseph Larmor (1857-1942) em 1897. No artigo intitulado On theory of the magnetic influence on spectra and the radiation from moving ions, Larmor (figura 1) apresentou sua expressão para o cálculo da potência irradiada por partículas carregadas [11].

A demonstração de Larmor permitiu explicar, por exemplo, a radiação térmica, que passou a ser analisada como o resultado da aceleração das cargas elétricas nas proximidades da superfície de um corpo em processo de agitação térmica. Ademais, a fórmula de Larmor foi decisivo, por exemplo, para o cálculo da intensidade das linhas espectrais do hidrogênio, para a evolução do modelo atômico e até mesmo para o embasamento da mecânica matricial [12].

Em 1898, Liénard (figura 1) apresentou o trabalho Champ électrique et magnétique produit par une charge électrique contentre é en un point et animée d'un mouvement quelconque [13], onde tanto demonstrou que o resultado obtido por Larmor só era válido para o limite não-relativístico como encontrou uma forma generalizada para a potência irradiada, sendo esta nova forma coerente em regimes relativísticos e não-relativísticos [14 15].

De fato, o interesse da comunidade científica sobre a radiação emitida por cargas aceleradas não findou após os resultados de Larmor e Liénard. Durante o século passado, inúmeros trabalhos científicos buscaram am-
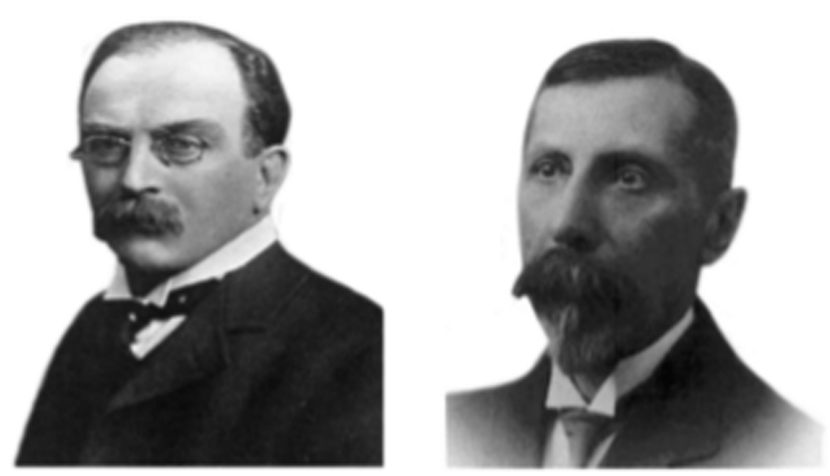

Figura 1: Joseph Larmor e Alfred-Marie Liénard (da esquerda para a direita). pliar a discussão sobre o referido fenômeno que, por sua vez, recebeu até mesmo atenção especial de renomados físicos como o alemão Max Born (1882-1970) [16 e o austro-norte-americano Wolfgang Pauli (1900-1958) 17. Dentre essas discussões, cita-se, por exemplo, a oposição de ideias sobre a radiação eletromagnética emitida por cargas uniformemente aceleradas, na qual, o próprio Pauli [17] além do físico alemão Max Von Laue (1879-1960) 18 alegavam que as cargas nessa condição de aceleração não emitiam radiação [19], enquanto o físico austro-britânico Hermann Bondi (1919-2005) e o astrofísico austríaco Thomas Gold (1920-2004) afirmavam o contrário 20]. Uma abordagem minuciosa desse problema foi desenvolvida posteriormente à luz da Teoria Clássica de Campos, em especial, com os trabalhos do físico austro-norte-americano Fritz Rohrlich (1921) 21,23$]$.

Com o desenvolvimento da teoria clássica da radiação muitos resultados experimentais puderam ser elucidados, dentre os quais, citam-se: o espalhamento de Rayleigh, o espalhamento de Thomson, o espalhamento de ressonância ou luminescência, o tão discutido problema da radiação térmica e os raios-x no efeito bremsstrahlung. O ulterior desenvolvimento da mecânica quântica e da relatividade conduziu a uma compreensão ainda mais elaborada dos fenômenos relacionados aos campos de radiação.

Inegavelmente a temática acerca dos fenômenos de radiação é hoje uma das mais produtivas da ciência moderna, estando presente em diversas áreas como astrofísica, biotecnologia, medicina nuclear, nanotecnologia, telecomunicações e computação quântica.

Neste artigo, usaremos a Teoria Clássica de Campos ${ }^{1}$ para tratar o campo eletromagnético e a interação de uma carga elétrica acelerada com esse no espaço-tempo de Minkowski.

Por razões didáticas, inicialmente, introduziremos as propriedades matemáticas do espaço-tempo em questão e obteremos a partir de uma formulação lagrangeana do campo eletromagnético, as equações do campo com fonte que, por sua vez, serão solucionadas pelo formalismo das funções de Green invariantes. De posse dessas soluções, encontraremos o tensor do campo eletromagnético e, após alguns passos, o padrão da radiação emitida pela carga acelerada (em condição não-relativística), a potência de Larmor e a generalização de Liénard.

Enfatiza-se que durante todo o texto, estaremos utilizando o sistema de unidades de Heaviside-Lorentz ${ }^{2}$ assumindo $c=1$.

\footnotetext{
1 Reforça-se que a abordagem via Teoria de Campos é atualmente onipresente para descrever as interações físicas não somente em condições clássicas, mas também em condições quânticas ou quânticorelativísticas.

2 Sistema de unidades eletromagnéticas que recebe esse nome em homenagem ao engenheiro e matemático inglês Oliver Heaviside (1850-1925) e ao físico holandês Hendrik Antoon Lorentz (18531928). Nesse sistema a constante elétrica $\epsilon_{0}$ e a constante magnética $\mu_{0}$ estão normalizadas $\left(\epsilon_{0}=1\right.$ e $\left.\mu_{0}=1\right)$ sendo substituídas pela constante $c$ nas equações de Maxwell. As unidades de HeavisideLorentz são racionalizadas, ou seja, não apresentam os fatores de
} 


\section{Espaço-tempo de Minkowski}

De modo geral, as peculiaridades de um determinado espaço-tempo podem ser obtidas através de seu elemento de linha que é invariante sob transformações características deste espaço-tempo, como é o caso, por exemplo, das transformações de Lorentz 26].

Considerando um espaço-tempo genérico quadridimensional, o seu respectivo elemento de linha pode ser expresso por

$$
d s^{2}=\sum_{\alpha=0}^{3} \sum_{\beta=0}^{3} g_{\alpha \beta}(x) d x^{\alpha} d x^{\beta},
$$

na qual, $g_{\alpha \beta}$ representa as componentes do tensor métrico do espaço-tempo.

Sabendo que o tensor métrico pode ser empregado para baixar e elevar índices e fazendo uso da soma de Einstein que omite o símbolo de somatório para índices repetidos, podemos reescrever a equação (1) no seguinte formato:

$$
d s^{2}=d x_{\beta} d x^{\beta} .
$$

Para um espaço-tempo arbitrário, o produto escalar entre dois vetores $A$ e $B$, por exemplo, é definido por

$$
\text { A. } B=A_{\nu} B^{\nu}=A^{\nu} B_{\nu} .
$$

Além do mais, a equação (3) quando escrita em termos da métrica resultará em:

$$
A_{\nu} B^{\nu}=g_{\mu \nu} A^{\mu} B^{\nu}=g^{\mu \nu} A_{\mu} B_{\nu}
$$

Nesse contexto, devem ser evocadas outras propriedades do tensor do espaço-métrico, a saber:

$$
\begin{gathered}
g_{\mu \nu} g^{\nu \alpha}=\delta_{\mu}^{\alpha} \\
g_{\alpha \beta}=\eta_{\alpha \beta}=\left[\begin{array}{rrrr}
1 & 0 & 0 & 0 \\
0 & -1 & 0 & 0 \\
0 & 0 & -1 & 0 \\
0 & 0 & 0 & -1
\end{array}\right]
\end{gathered}
$$

onde $g_{\alpha \beta}$ é denominado de métrica de Minkowski.

Com base nessas últimas relações, o elemento de linha pode ser reescrito como

$$
d s^{2}=d t^{2}-d \vec{r}^{2}
$$

onde, $d t=d x^{0}, d x=d x^{1}, d y=d x^{2}, d z=d x^{3}$ e $d \vec{r}=$ $\hat{x} d x+\hat{y} d y+\hat{z} d z$.

Por fim, os operadores diferenciais no espaço quadridimensional são definidos como

$$
\frac{\partial}{\partial x^{\mu}}=\partial_{\mu} \quad, \quad \frac{\partial}{\partial x_{\mu}}=\partial^{\mu},
$$

$4 \pi$ e são comumente usadas no âmbito da física das partículas elementares e campos 24,25. e o operador d'Alembertiano como

$$
\partial^{\mu} \partial_{\mu}=\partial_{\mu} \partial^{\mu}=\frac{\partial^{2}}{\partial t^{2}}-\nabla^{2}
$$

\section{Obtenção das equações de Maxwell inomogêneas via teoria clássica de campos}

De imediato, seja uma ação clássica $S$ definida por

$$
S=\int £\left(\xi, \partial^{\mu} \xi\right) d^{4} x
$$

na qual, $£$ representa a densidade lagrangeana dependente de um parâmetro covariante $\xi\left(x^{\mu}\right)$ e de sua derivada $\partial_{\mu} \xi$.

Para sistemas contínuos, o princípio variacional estabelece que 27:

$$
\delta S=\delta \int £\left(\xi, \partial^{\mu} \xi\right) d^{4} x=0 .
$$

Admitindo que o quadripotencial do campo eletromagnético $A_{\nu}$ seja o parâmetro covariante, a equação 10 resultará em:

$$
\delta S=\int \delta £\left(A_{\nu}, \partial_{\mu} A_{\nu}\right) d^{4} x=0 .
$$

Desenvolvendo a equação (11), como se segue

$$
\begin{gathered}
\int\left[\frac{\partial £}{\partial A_{\nu}} \delta A_{\nu}+\frac{\partial £}{\partial\left(\frac{\partial A_{\nu}}{\partial x^{\mu}}\right)} \delta\left(\partial_{\mu} A_{\nu}\right)\right] d^{4} x=0 \Longrightarrow \\
\int \frac{\partial £}{\partial A_{\nu}} \delta A_{\nu} d^{4} x+\int \frac{\partial £}{\partial\left(\partial_{\mu} A_{\nu}\right)} \partial_{\mu}\left(\delta A_{\nu}\right) d^{4} x=0
\end{gathered}
$$

alcançaremos a seguinte expressão:

$$
\begin{aligned}
& {\left[\frac{\partial £}{\partial\left(\partial_{\mu} A_{\nu}\right)} \delta A_{\nu}\right]_{x_{2}^{\mu}}^{x_{1}^{\mu}}-\int \partial_{\mu}\left(\frac{\partial £}{\partial\left(\partial_{\mu} A_{\nu}\right)}\right) \delta A_{\nu} d^{4} x+} \\
& \int \frac{\partial £}{\partial A_{\nu}} \delta A_{\nu} d^{4} x=0
\end{aligned}
$$

Além disso, sabendo que em pontos extremos $\delta A_{\nu}\left(x_{1}^{\mu}\right)=$ $\delta A_{\nu}\left(x_{2}^{\mu}\right)=0$ e fazendo uso dessa relação na equação 12 , obtemos

$$
\begin{gathered}
\int\left[\frac{\partial £}{\partial A_{\nu}}-\frac{\partial}{\partial x^{\mu}}\left(\frac{\partial £}{\partial\left(\frac{\partial A_{\nu}}{\partial x^{\mu}}\right)}\right)\right] \delta A_{\nu}=0 \Longrightarrow \\
\frac{\partial £}{\partial A_{\nu}}-\frac{\partial}{\partial x^{\mu}}\left(\frac{\partial £}{\partial\left(\frac{\partial A_{\nu}}{\partial x^{\mu}}\right)}\right)=0
\end{gathered}
$$

que é a forma covariante das equações de Euler-Lagrange. 
Definindo $\frac{\partial A_{\nu}}{\partial x^{\mu}}=\partial_{\mu} A_{\nu}$ e substituindo essa relação na equação 13 , teremos:

$$
\frac{\partial £}{\partial A_{\nu}}=\partial_{\mu}\left(\frac{\partial £}{\partial\left(\partial_{\mu} A_{\nu}\right)}\right)
$$

Com base nas características da lagrangeana para sistemas discretos, é razoável que na ausência de cargas, a lagrangeana (equação 14) apresente termos quadráticos nas velocidades generalizadas $\partial_{\mu} A_{\nu}[28$. Para atender essas características, pode-se utilizar um escalar de Lorentz que seja simultaneamente invariante por inversão e transformações de Lorentz próprias e ortócronas. Nesse sentido, empregaremos o escalar dado pelas seguintes relações [29]:

$$
\begin{gathered}
F^{\alpha \beta} F_{\alpha \beta}=2\left(B^{2}-E^{2}\right) \\
F^{\alpha \beta}=\eta^{\alpha \gamma} F_{\gamma \rho} \eta^{\rho \beta}
\end{gathered}
$$

Onde:

$$
F_{\alpha \beta}=\left[\begin{array}{cccc}
0 & E_{x} & E_{y} & E_{z} \\
-E_{x} & 0 & -B_{z} & B_{y} \\
-E_{y} & B_{z} & 0 & -B_{x} \\
-E_{z} & -B_{y} & B_{x} & 0
\end{array}\right]
$$

As expressões 15 e (16) são, nessa ordem, as componentes variantes e contravariantes do tensor de ordem dois que representa o campo, enquanto que as ternas $\left(E_{x}\right.$, $\left.E_{y}, E_{z}\right)$ e $\left(B_{x}, B_{y}, B_{z}\right)$ correspondem às componentes do campo elétrico e magnético, respectivamente.

No que se refere ao termo de interação da lagrangeana, pode-se admitir o escalar de Lorentz $J^{\alpha} A_{\alpha}$, haja vista que este apresenta acoplamento mínimo entre a quadricorrente $\left(J^{\alpha}\right)$ e o quadripotencial do campo $\left(A_{\alpha}\right)$.

Baseado nessas motivações, podemos utilizar uma densidade de lagrangeana do tipo:

$$
£=-\frac{1}{4} F^{\alpha \beta} F_{\alpha \beta}-J^{\alpha} A_{\alpha} .
$$

Sendo o tensor de ordem dois (que representa o campo eletromagnético) dado pela expressão

$$
F^{\alpha \beta}=\partial^{\alpha} A^{\beta}-\partial^{\beta} A^{\alpha}
$$

e substituindo esse tensor na equação (17), obtemos a densidade de lagrangeana dada por

$£=-\frac{1}{4} \eta_{\lambda \alpha} \eta_{\sigma \beta}\left(\partial^{\alpha} A^{\sigma}-\partial^{\sigma} A^{\alpha}\right)\left(\partial^{\lambda} A^{\beta}-\partial^{\beta} A^{\lambda}\right)-J^{\alpha} A_{\alpha}$.

Aplicando as relações de Euler-Lagrange, alcançamos a relação

$$
\frac{\partial £}{\partial A_{\nu}}=-J^{\alpha} \frac{\partial A_{\alpha}}{\partial A_{\nu}}=-J^{\alpha} \delta_{\nu \alpha}=-J^{\nu}
$$

e após minuciosos passos, obtemos a equação 21.

$$
\begin{aligned}
& \frac{\partial £}{\partial\left(\partial_{\mu} A_{\nu}\right)}=-\frac{1}{4} \eta_{\lambda \alpha} \eta_{\sigma \beta}\left\{\left[\frac{\partial\left(\partial^{\alpha} A^{\sigma}\right)}{\partial\left(\partial_{\mu} A_{\nu}\right)}-\frac{\partial\left(\partial^{\sigma} A^{\alpha}\right)}{\partial\left(\partial_{\mu} A_{\nu}\right)}\right] F^{\lambda \sigma}+\left[\frac{\partial\left(\partial^{\lambda} A^{\sigma}\right)}{\partial\left(\partial_{\mu} A_{\nu}\right)}-\frac{\partial\left(\partial^{\sigma} A^{\lambda}\right)}{\partial\left(\partial_{\mu} A_{\nu}\right)}\right] F^{\alpha \beta}\right\} \quad \therefore \\
& \frac{\partial \notin}{\partial\left(\partial_{\mu} A_{\nu}\right)}=-\frac{1}{4}\left\{\left[\frac{\partial\left(\partial_{\alpha} A_{\sigma}\right)}{\partial\left(\partial_{\mu} A_{\nu}\right)}-\frac{\partial\left(\partial_{\sigma} A_{\alpha}\right)}{\partial\left(\partial_{\mu} A_{\nu}\right)}\right] F^{\lambda \sigma}+\left[\frac{\partial\left(\partial_{\lambda} A_{\sigma}\right)}{\partial\left(\partial_{\mu} A_{\nu}\right)}-\frac{\partial\left(\partial_{\sigma} A_{\lambda}\right)}{\partial\left(\partial_{\mu} A_{\nu}\right)}\right] F^{\alpha \beta}\right\} \quad \therefore \\
& \frac{\partial £}{\partial\left(\partial_{\mu} A_{\nu}\right)}=-\frac{1}{4}\left[\left(\delta_{\lambda \mu} \delta_{\sigma \nu}-\delta_{\sigma \mu} \delta_{\sigma \nu}\right) F^{\lambda \sigma}+\left(\delta_{\lambda \mu} \delta_{\sigma \nu}-\delta_{\sigma \mu} \delta_{\lambda \nu}\right) F^{\alpha \beta}\right] \quad \therefore \\
& \frac{\partial £}{\partial\left(\partial_{\mu} A_{\nu}\right)}=-F^{\mu \nu}
\end{aligned}
$$

Se substituirmos essas duas últimas relações 20 e 21) na equação (14), alcançamos a forma covariante das equações de Maxwell não-homogêneas expressa por

$$
\partial_{\mu} F^{\mu \nu}=J^{\nu}
$$

que, por sua vez, representa quatro do conjunto de oito equações.

As quatro outras equações (homogêneas) surgem da identidade tensorial [30], dada por

$$
\partial^{\alpha} F^{\beta \gamma}+\partial^{\beta} F^{\gamma \alpha}+\partial^{\gamma} F^{\alpha \beta}=0
$$

e decorrem da anti-simetria do tensor de Maxwell $\left(F^{\mu \nu}=\right.$ $\left.-F^{\nu \mu}\right)$. Substituindo a definição de tensor do campo eletromagnético (equação 18) na expressão (22), obtemos:

$$
\partial_{\mu} \partial^{\mu} A^{\nu}-\partial^{\nu}\left(\partial_{\mu} A^{\mu}\right)=J^{\nu}
$$

De fato, uma vez que o quadripotencial $A^{\mu}$ satisfizer a condição de Lorenz $\left(\partial_{\mu} A^{\mu}=0\right)$, este, por consequência, será uma solução da equação de onda quadridimensional, ou seja:

$$
\partial_{\mu} \partial^{\mu} A^{\nu}=J^{\nu}
$$




\section{Soluções das equações de campo pelo formalismo das funções de Green invariantes}

Nessa seção, a solução da equação 25 será desenvolvida mediante o emprego da função de Green invariante ou propagador. Para tal propósito, o primeiro passo consiste na aplicação da seguinte expressão

$$
\left(\partial^{\mu} \partial_{\mu}\right)_{x} D\left(x, x^{\prime}\right)=\delta^{(4)}\left(x-x^{\prime}\right)
$$

na qual, $D\left(x, x^{\prime}\right)$ consiste na função de Green ou propagador, $\left(\partial^{\mu} \partial_{\mu}\right)_{x}$ indica o operador d' alembertiano atuando na coordenada $x$ em que $x^{\mu}=\left(x^{0}, x^{1}, x^{2}, x^{3}\right)$, e $\delta^{(4)}\left(x-x^{\prime}\right)$ é a função delta de Dirac quadridimensional.

Sabe-se que uma relevante propriedade da função de Green do operador d' Alembertiano é que esta, na ausência de superfícies de contorno, depende unicamente da diferença entre os vetores posição espaço-temporais $x-x^{\prime}=\zeta 29$, onde definimos o intervalo espacial $\zeta^{i}=x^{i}-x^{i^{\prime}}$ como $R^{i}$, isto é, $\zeta^{i}=R^{i}$. Nesse sentido, devemos realizar uma transformação de variável a fim de que o operador $\partial_{\mu} \partial^{\mu}$ atue na variável $\zeta$. Desse modo, fazemos

$$
\frac{\partial}{\partial x^{\mu}}=\frac{\partial}{\partial \zeta^{\nu}} \frac{\partial \zeta^{\nu}}{\partial x^{\mu}}=\delta_{\mu}^{\nu} \frac{\partial}{\partial \zeta^{\nu}}=\frac{\partial}{\partial \zeta^{\mu}}
$$

e, por conseguinte

$$
\left[\partial_{\mu} \partial^{\mu}\right]_{x}=\left[\partial_{\mu} \partial^{\mu}\right]_{\zeta}
$$

Observa-se que nesse caso, $D\left(x, x^{\prime}\right)=D\left(x-x^{\prime}\right)=$ $D(\zeta)$, o que nos permite reescrever a equação 26 como:

$$
\left[\partial_{\mu} \partial^{\mu}\right]_{\zeta}\left(D\left(x, x^{\prime}\right)\right)=\delta(\zeta)
$$

O próximo passo consiste em usar a integral de Fourier para transformar do espaço de coordenadas para o espaço do número de onda (ou espaço dos momentos). Em detalhe, a transformada de Fourier $\tilde{D}(k)$ pode ser definida por

$$
D(\zeta)=\frac{1}{16 \pi^{4}} \int \tilde{D}(k) e^{-i k_{\nu} \zeta^{\nu}} d^{4} k
$$

onde o argumento da exponencial é dado por:

$$
K_{\mu} \zeta^{\mu}=\eta_{\nu \mu} k^{\nu} k^{\mu}=k^{0} \zeta^{0}-|\vec{k}| R .
$$

A função delta, por sua vez, pode ser representada por:

$$
\delta(\zeta)=\frac{1}{16 \pi^{4}} \int e^{-i k_{\nu} \zeta^{\nu}} d^{4} k .
$$

Substituindo adequadamente a expressão 28 em 27 e aplicando o operador $\left(\partial^{\mu} \partial_{\mu}\right)_{\zeta}$, alcançamos

$$
\left(\partial^{\alpha} \partial_{\alpha}\right)_{\zeta} D(\zeta)=\frac{1}{16 \pi^{4}} \int \tilde{D}(k)\left(\partial^{\alpha} \partial_{\alpha}\right)_{\zeta} e^{-i k_{\nu} \zeta^{\nu}} d^{4} k
$$

Analisando minuciosamente a aplicação do operador,

$$
\begin{gathered}
\left(\partial^{\mu} \partial_{\mu}\right)_{\zeta} e^{-i \eta_{\alpha \nu} k^{\alpha} \zeta^{\nu}}=\partial^{\mu}\left[-i \eta_{\alpha \nu} K^{\alpha} \frac{\partial \zeta^{\nu}}{\partial \zeta^{\mu}} e^{-i \eta_{\beta \nu} k^{\beta} \zeta^{\nu}}\right] \\
\left(\partial^{\mu} \partial_{\mu}\right)_{\zeta} e^{-i \eta_{\alpha \nu} k^{\alpha} \zeta^{\nu}}=\partial^{\mu}\left[-i \eta_{\alpha \nu} k^{\alpha} e^{-i \eta^{\beta \nu} k_{\beta} \zeta_{\nu}}\right] \\
\left(\partial^{\mu} \partial_{\mu}\right)_{\zeta} e^{-i \eta_{\alpha \nu} k^{\alpha} \zeta^{\nu}}=-\eta_{\alpha \mu} \eta^{\beta \nu} k^{\alpha} k_{\beta} \delta_{\nu \mu} e^{-i \eta^{\beta \nu} k_{\beta} k_{\nu}} \\
\left(\partial^{\mu} \partial_{\mu}\right)_{\zeta} e^{-i \eta_{\alpha \nu} k^{\alpha} \zeta^{\nu}}=-\eta_{\alpha \nu} \eta^{\beta \nu} k^{\alpha} k_{\beta} e^{-i \eta^{\beta \nu} k_{\beta} \zeta_{\nu}} \\
\left(\partial^{\mu} \partial_{\mu}\right)_{\zeta} e^{-i \eta_{\alpha \nu} k^{\alpha} \zeta^{\nu}}=-\delta_{\alpha}^{\beta} k^{\alpha} k_{\beta} e^{-i \eta^{\beta \nu} k_{\beta} \zeta_{\nu}}
\end{gathered}
$$

obtemos a seguinte expressão:

$$
\left(\partial^{\mu} \partial_{\mu}\right)_{\zeta} e^{-i \eta_{\alpha \nu} k^{\alpha} \zeta^{\nu}}=-k^{\alpha} k_{\alpha} e^{-i k^{\alpha} \zeta_{\alpha}} .
$$

Substituindo a expressão 31 em 30, temos:

$$
\tilde{D}(k)=\frac{i^{2}}{k^{\alpha} k_{\alpha}} .
$$

Por sua vez, alocando essa última equação (32) na transformada de Fourier da função de Green (28), obtemos:

$$
D(\zeta)=\frac{i^{2}}{16 \pi^{4}} \int \frac{e^{-i k_{\nu} \zeta^{\nu}}}{k^{\alpha} k_{\alpha}} d^{4} k .
$$

Sabendo que $d^{4} k=d k^{0} d^{3} k$ e usando a seguinte relação

$$
k^{\alpha} K_{\alpha}=\left(k^{0}\right)^{2}-|\vec{k}|^{2}
$$

podemos reescrever a função de Green invariante ou propagador como

$$
D(\zeta)=-\frac{1}{16 \pi^{4}} \int e^{i \vec{k} \vec{R}} d^{3} k \int_{-\infty}^{+\infty} \frac{e^{-i k^{0} \zeta^{0}}}{\left[\left(k^{0}\right)^{2}-|\vec{k}|^{2}\right]} d k^{0}
$$

onde o integrando em $d k^{0}$ não está definido para $k^{0}=$ $\pm|\vec{k}|$.

Nesse ínterim, atentaremos à solução da integral em $k^{0}$, isto é:

$$
\int_{-\infty}^{+\infty} \frac{e^{-i k^{0} \zeta^{0}}}{\left[\left(k^{0}\right)^{2}-k^{i} k^{i}\right]} d k^{0}
$$

Em detalhe, o quadrivetor $k^{\mu}$ é representado em termos de suas componentes, $k=(\omega, \vec{k})$, onde $\omega$ consiste na frequência e $\vec{k}$ é o vetor de onda. Convém mencionar que $\omega$ e $\vec{k}$ estão relacionados à energia e ao momento linear da onda, respectivamente.

Em razão do integrando 36 apresentar pontos de singularidade (figura 2), empregaremos a teoria de resíduos e polos para solucionar a integral em $d k^{0}$. Embasado nessa teoria, devemos considerar a integral em $k^{0}$ como uma variável complexa e resolver a integral resultante como uma integral de contorno no plano complexo $k^{0}$. Conforme ilustra a figura 2, o integrando possui dois polos simples, a saber: 


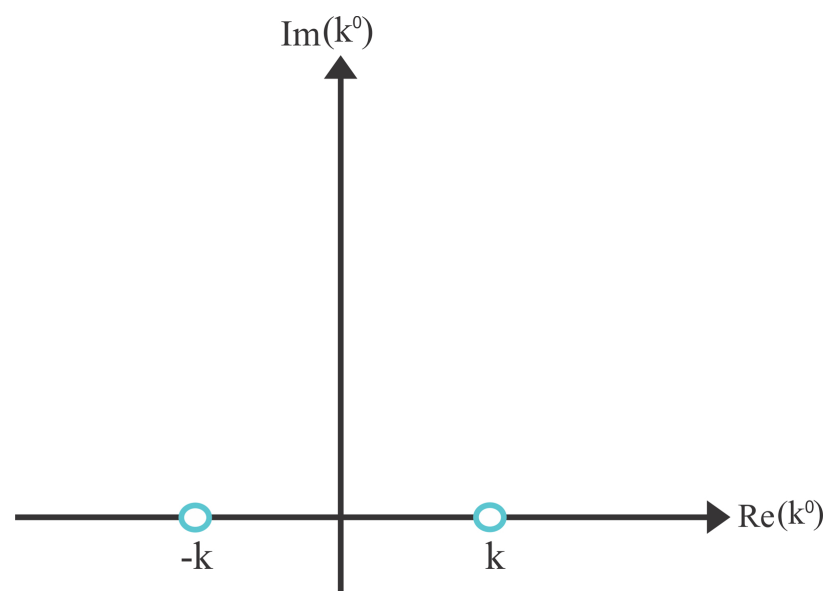

Figura 2: Os pontos de singularidade do integrando da expressão (36) dispostos no eixo real $k^{0}$.

$$
k^{0}= \pm\left(k^{i} k^{i}\right)^{\frac{1}{2}}= \pm|\vec{k}|
$$

As soluções distintas das funções de Green (26) podem ser obtidas tomando os contornos fechados $r$ e $a$ e deslocando os polos no eixo imaginário de uma quantidade $-\varepsilon$ para o contorno $r$ ou $+\varepsilon$ para o contorno $a$ conforme ilustram as figuras $3 \mathrm{e} 4$. Por fim, tomamos o limite $\varepsilon \rightarrow 0$.

O contorno $r$, caracterizado pela fronteira de um semicírculo de raio $R$ definido no semiplano inferior e contendo os polos $k^{0}= \pm|\vec{k}|-i \varepsilon$, deve ser selecionado quando $\zeta^{0}>0$, pois o termo $e^{-i k^{0} \zeta^{0}}$ diverge no semiplano superior quando $R \rightarrow \infty$. Por outro lado, o contorno $a$, caracterizado pela fronteira de um semicírculo de raio $R$ traçado no semiplano superior e abarcando os polos $k^{0}= \pm|\vec{k}|+i \varepsilon$, deve ser selecionado quando $\zeta^{0}<0$, pelo fato de $e^{-i k^{0} \zeta^{0}}$ divergir no semiplano inferior quando $R \rightarrow \infty$. Para demonstrarmos essas divergências, consideremos as seguintes expressões:

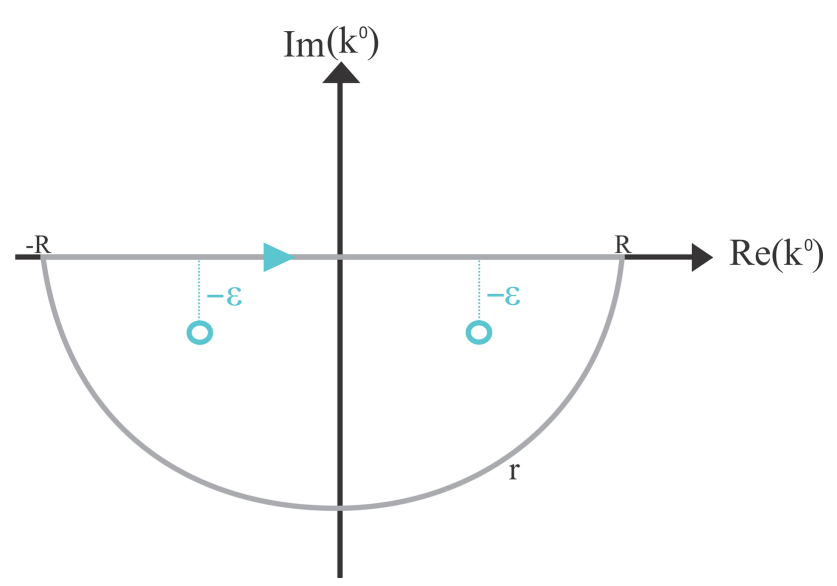

Figura 3: Os polos deslocados no eixo imaginário de uma quantidade $-\epsilon$.

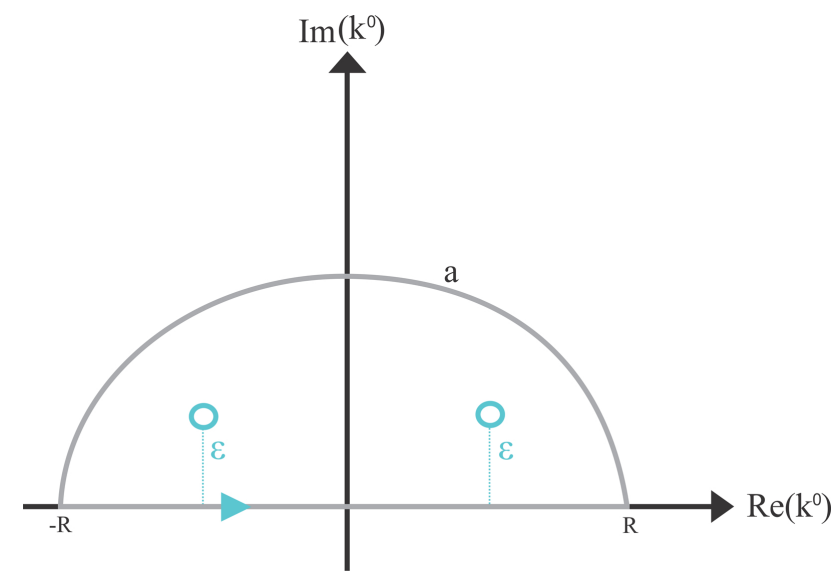

Figura 4: Os polos deslocados no eixo imaginário de uma quantidade $+\epsilon$.

$$
\begin{gathered}
k^{0}=a+b i \\
e^{-i k^{0} \zeta^{0}}=e^{-i a \zeta^{0}} e^{b \zeta^{0}}
\end{gathered}
$$

Com base na equação 39 , observa-se que se $\zeta^{0}>0$ e $b \rightarrow \infty$, o termo $e^{b \zeta^{0}} \rightarrow \infty$, o que acarreta a divergência no semiplano superior. Por outro lado, se $\zeta^{0}<0$ e $b \rightarrow$ $-\infty$, o termo $e^{b \zeta^{0}} \rightarrow \infty$, promovendo assim a divergência no semiplano inferior.

Aplicando a teoria dos resíduos e polos 31] na equação (36) e assumindo o contorno fechado $r$, temos:

$$
\int_{r} \frac{e^{-i k^{0} \zeta^{0}}}{\left[\left(k^{0}\right)^{2}-|\vec{k}|^{2}\right]} d k^{0}=-2 \pi i \sum r e s .
$$

Reescrevendo a integral $(36)$ como a soma da integral sobre o eixo real de $k^{0}$ e da integral sobre a semicircunferência de raio $R$, obtém-se a expressão:

$$
\begin{aligned}
& \int_{-R}^{+R} \frac{e^{-i k^{0} \zeta^{0}}}{\left[\left(k^{0}\right)^{2}-|\vec{k}|^{2}\right]} d k^{0}+\int_{C_{1}} \frac{e^{-i k^{0} \zeta^{0}}}{\left[\left(k^{0}\right)^{2}-|\vec{k}|^{2}\right]} d k^{0} \\
& =-2 \pi i \sum \text { res }
\end{aligned}
$$

Admitindo que $R \rightarrow \infty$ e que o somatório dos resíduos não depende de $R$, alcançamos a seguinte equação:

$$
\lim _{R \rightarrow \infty}\left[\int_{C_{1}} f\left(k^{0}\right) d k^{0}\right]+\int_{-\infty}^{+\infty} f\left(k^{0}\right) d k^{0}=-2 \pi i \sum r e s .
$$

Para obter o valor real da integral no contorno $C_{1}$, devemos tratar a desigualdade

$$
\left|\int_{c} f(\zeta) d \zeta\right| \leq \int_{c}|f(\zeta)||d \zeta|
$$


onde $k^{0}=R e^{i \alpha}$ e $\alpha \in[\pi, 2 \pi]$.

Sabendo que $\int_{c} f(\zeta) d \zeta$ para o contorno $C_{1}$ é dada por

$$
\int_{C_{1}} \frac{e^{-i k^{0} \zeta^{0}}}{\left[\left(k^{0}\right)^{2}-|\vec{k}|^{2}\right]} d k^{0}=\int_{C_{1}} i R \frac{e^{i \alpha} e^{-i R \zeta^{0} e^{i \alpha}}}{R^{2} e^{2 i \alpha}-|\vec{k}|^{2}} d \alpha
$$

definindo $I$ como

$$
I=i R \frac{e^{i \alpha} e^{-i R \zeta^{0} e^{i \alpha}}}{\left[R^{2} e^{2 i \alpha}-|\vec{k}|^{2}\right]}
$$

e usando a desigualdade 42 para tratar a integral do segundo membro da equação (43), obtemos:

$$
\left|\int_{\pi}^{2 \pi} I d \alpha\right| \leq \frac{R}{\left[R^{2}-|\vec{k}|^{2}\right]} \int_{\pi}^{2 \pi} e^{R \zeta^{0} \operatorname{sen}(\alpha)} d \alpha
$$

Uma vez que $\zeta^{0}>0$ e $\operatorname{sen}(\alpha)<0$, então, $\zeta^{0} \operatorname{sen}(\alpha)<0$. Ademais, admitindo que $R \rightarrow \infty$, os termos $e^{R \zeta^{0} \operatorname{sen}(\alpha)} \mathrm{e}$ $\frac{R}{R^{2}-|\vec{k}|^{2}}$ tendem a zero. Essas observações nos permitem verificar que

$$
\lim _{R \rightarrow \infty}\left\{\int_{C_{1}} \frac{e^{-i k^{0} \zeta^{0}}}{\left[\left(k^{0}\right)^{2}-|\vec{k}|^{2}\right]} d k^{0}\right\}=0,
$$

e, por consequência:

$$
\int_{-\infty}^{+\infty} \frac{e^{-i k^{0} \zeta^{0}}}{\left[\left(k^{0}\right)^{2}-|\vec{k}|^{2}\right]} d k^{0}=-2 \pi i \sum \text { res } .
$$

Desenvolvendo o somatório dos resíduos

$$
\begin{aligned}
\sum r e s= & \lim _{k^{0} \rightarrow(|\vec{k}|-i \varepsilon)}\left\{\left[\frac{k^{0}-(|\vec{k}|-i \varepsilon)}{k^{0}-(|\vec{k}|-i \varepsilon)}\right]\left[\frac{e^{-i k^{0} \zeta^{0}}}{k^{0}-(-|\vec{k}|-i \varepsilon)}\right]\right\}+ \\
& \lim _{k^{0} \rightarrow(-|\vec{k}|-i \varepsilon)}\left\{\left[\frac{k^{0}-(-|\vec{k}|-i \varepsilon)}{k^{0}-(|\vec{k}|-i \varepsilon)}\right]\left[\frac{e^{-i k^{0} \zeta^{0}}}{k^{0}-(-|\vec{k}|-i \varepsilon)}\right]\right\}
\end{aligned}
$$

alcançamos a relação:

$$
\sum r e s=\frac{1}{2|\vec{k}|}\left\{e^{-i \zeta^{0}(|\vec{k}|-i \varepsilon)}-e^{-i \zeta^{0}(-|\vec{k}|-i \varepsilon)}\right\}
$$

Tomando $\varepsilon$ tão pequeno quanto se deseja, temos:

$$
\begin{gathered}
\lim _{\varepsilon \rightarrow 0} \sum r e s=\frac{1}{2|\vec{k}|}\left[e^{-i|\vec{k}| \zeta^{0}}-e^{i|\vec{k}| \zeta^{0}}\right] \\
\int_{-\infty}^{+\infty} \frac{e^{-i k^{0} \zeta^{0}}}{\left(k^{0}\right)^{2}-|\vec{k}|^{2}} d k^{0}=\frac{\pi i}{|\vec{k}|}\left[e^{i|\vec{k}| \zeta^{0}}-e^{-i|\vec{k}| \zeta^{0}}\right]
\end{gathered}
$$

De modo análogo, empregaremos a teoria de resíduos e polos para solucionar a integral em $d k^{0}$ da equação 35, arbitrando o contorno fechado $a$. Desse modo:

$$
\int_{a} \frac{e^{-i k^{0} \zeta^{0}}}{\left[\left(k^{0}\right)^{2}-|\vec{k}|^{2}\right]} d k^{0}=2 \pi i \sum r e s .
$$

Separando adequadamente a integral fechada em um integral sobre o eixo real de $k^{0}$ e outra sobre a semicircunferência do círculo de raio $R$ no semiplano superior de $k^{0}$, ou seja,

$$
\int_{a} f\left(k^{0}\right) d k^{0}=\int_{C_{2}} f\left(k^{0}\right) d k^{0}+\int_{-R}^{+R} f\left(k^{0}\right) d k^{0}
$$

e tomando o limite em que $R \rightarrow \infty$, alcançamos:

$\lim _{R \rightarrow \infty}\left[\int_{C_{2}} f\left(k^{0}\right) d k^{0}\right]+\int_{-\infty}^{+\infty} f\left(k^{0}\right) d k^{0}=2 \pi i \sum r e s$. (52)

Convém observar que o somatório dos resíduos não depende de $R$. Nesse caso, aplicando a relação (46), temos

$$
\int_{-\infty}^{+\infty} \frac{e^{-i k^{0} \zeta^{0}}}{\left[\left(k^{0}\right)^{2}-|\vec{k}|^{2}\right]} d k^{0}=2 \pi i \sum r e s
$$

na qual, a somatória dos resíduos é dada por

$$
\sum r e s=\frac{1}{2|\vec{k}|}\left\{e^{-i \zeta^{0}(|\vec{k}|+i \varepsilon)}-e^{-i \zeta^{0}(-|\vec{k}|+i \varepsilon)}\right\} .
$$

Novamente, admitindo $\varepsilon \rightarrow 0$, a expressão 53 , tornase-á:

$$
\int_{-\infty}^{+\infty} \frac{e^{-i k^{0} \zeta^{0}}}{\left(k^{0}\right)^{2}-|\vec{k}|^{2}} d k^{0}=\frac{\pi i}{|\vec{k}|}\left[e^{-i|\vec{k}| \zeta^{0}}-e^{i|\vec{k}| \zeta^{0}}\right] .
$$

Substituindo a expressão 49 em 35 , alcançamos a função de Green retardada ou causal, dada por:

$$
D_{\text {ret }}(\zeta)=\frac{\theta\left(\zeta^{0}\right)}{8 \pi^{3}} \int \frac{\operatorname{sen}\left(|\vec{k}| \zeta^{0}\right) e^{i \vec{k} \vec{R}}}{|\vec{k}|} d^{3}|\vec{k}|
$$

Nesse caso, a função de Heaviside $\theta\left(\zeta^{0}\right)$ surge em decorrência do fato de assumirmos um contorno fechado $r$ onde $\zeta^{0}>0$. Sabendo que a função de Heaviside é definida como

$$
\theta\left(\zeta^{0}\right)= \begin{cases}1, & \text { se } \quad \zeta^{0}>0 \\ 0, & \text { se } \quad \zeta^{0}<0\end{cases}
$$

e que em coordenadas esféricas

$$
d^{3} \vec{k}=|\vec{k}|^{2} \operatorname{sen}(\theta) d \theta d \phi d|\vec{k}|
$$

então, a equação 56 pode ser reescrita como: 


$$
D_{\text {res }}(\zeta)=\frac{\theta\left(\zeta^{0}\right)}{2 \pi^{2} R} \int_{0}^{+\infty} \operatorname{sen}(|\vec{k}| R) \operatorname{sen}\left(|\vec{k}| \zeta^{0}\right) d|\vec{k}|
$$

Na equação (57), $R$ refere-se à distância espacial entre $x^{\mu}$ e $x^{\prime \mu}$. Reescrevendo as funções trigonométricas em termos de exponencias complexas, isto é,

$$
\begin{aligned}
\operatorname{sen}(|\vec{k}| R) \operatorname{sen}\left(|\vec{k}| \zeta^{0}\right) & =\frac{1}{4}\left[e^{i|\vec{k}|\left(\zeta^{0}-R\right)}+e^{-i|\vec{k}|\left(\zeta^{0}-R\right)}\right] \\
& +\frac{1}{4}\left[e^{i|\vec{k}|\left(\zeta^{0}+R\right)}+e^{-i|\vec{k}|\left(\zeta^{0}+R\right)}\right]
\end{aligned}
$$

integrando convenientemente as exponenciais conforme apresentado abaixo

$$
\begin{aligned}
& \int_{0}^{+\infty} e^{i|\vec{k}|\left(R-\zeta^{0}\right)} d|\vec{k}|=\int_{-\infty}^{0} e^{i|\vec{k}|\left(\zeta^{0}-R\right)} d|\vec{k}| \\
& \int_{0}^{+\infty} e^{-i|\vec{k}|\left(\zeta^{0}+R\right)} d|\vec{k}|=\int_{-\infty}^{0} e^{i|\vec{k}|\left(\zeta^{0}+R\right)} d|\vec{k}|
\end{aligned}
$$

e substituindo apropriadamente essas expressões 58,59 e 60 na expressão (57), alcançamos:

$$
D_{r e t}(\zeta)=\frac{\theta\left(\zeta^{0}\right)}{8 \pi^{2} R} \int_{-\infty}^{+\infty}\left[e^{i|\vec{k}|\left(\zeta^{0}-R\right)}-e^{i|\vec{k}|\left(\zeta^{0}+R\right)}\right] d|\vec{k}| .
$$

Aplicando a definição do delta de Dirac em termos do vetor de onda, isto é

$$
\delta\left(\zeta^{0} \pm R\right)=\frac{1}{2 \pi} \int_{-\infty}^{+\infty} e^{i|\vec{k}|\left(\zeta^{0} \pm R\right)} d|\vec{k}|
$$

a expressão 61, torna-se:

$$
D_{\text {ret }}(\zeta)=\frac{1}{4 \pi R} \theta\left(x^{0}-x^{\prime 0}\right) \delta\left(x^{0}-x^{\prime 0}-R\right) .
$$

Desenvolvendo um tratamento similar ao que fora realizado para a função $D_{\text {ret }}$, porém fazendo a integral sobre o contorno fechado $a$ e observando através da desigualdade 42 que a integral se anula no contorno $C_{2}$, temos:

$D_{a \nu}(\zeta)=\frac{\theta\left(\zeta^{\prime 0}\right)}{2 \pi^{2} R} \int_{-\infty}^{+\infty}\left[e^{i|\vec{k}|\left(\zeta^{\prime 0}-R\right)}-e^{i|\vec{k}|\left(\zeta^{\prime 0}+R\right)}\right] d|\vec{k}|$.

Aplicando a relação 62 em 64, alcançamos:

$$
D_{a \nu}(\zeta)=\frac{\theta\left(\zeta^{\prime} 0\right)}{4 \pi R}\left[\delta\left(\zeta^{\prime 0}-R\right)-\delta\left(\zeta^{\prime 0}+R\right)\right]
$$

Uma vez que $\zeta^{\prime} 0=-\zeta^{0}$ e $\delta\left[-\left(\zeta^{0}+R\right)\right]=\delta\left(\zeta^{0}+R\right)$, a equação 65 resultará em

$$
D_{a \nu}(\zeta)=\frac{1}{4 \pi R} \theta\left(-\zeta^{0}\right) \delta\left(\zeta^{0}+R\right)
$$

na qual, a função de Heaviside figura em decorrência da condição de contorno $a$, onde $\zeta^{0}<0$.

Sabendo que as funções de Green podem ser dispostas em uma forma covariante a partir da seguinte propriedade do delta de Dirac:

$$
\delta[f(x)]=\sum_{i} \delta\left(x-x_{i}\right)\left|\frac{d f\left(x_{i}\right)}{d x}\right|^{-1} .
$$

E que nesse caso

$$
\delta\left[\left(x^{\mu}-x^{\prime \mu}\right)^{2}\right]=\delta\left[\zeta^{\mu} \zeta_{\mu}\right]=\delta\left[\left(\zeta^{0}\right)^{2}-R^{2}\right]
$$

se diferenciarmos $\delta\left[\left(\zeta^{0}\right)^{2}-R^{2}\right]$ em relação a $z^{0}$ e realizarmos as devidas manipulações algébricas, teremos:

$$
\begin{aligned}
\delta\left[\left(\zeta^{0}\right)^{2}-R^{2}\right] & =\frac{1}{2 R}\left[\delta\left(x^{0}-x^{\prime 0}-R\right)\right. \\
& \left.+\delta\left(x^{0}-x^{\prime 0}+R\right)\right]
\end{aligned}
$$

Dessa forma, observa-se que, uma vez que as funções teta selecionam um ou outro entre os dois termos da expressão 69, , então:

$$
\begin{aligned}
& D_{r e t}(\zeta)=\frac{1}{2 \pi} \theta\left(x^{0}-x^{\prime 0}\right) \delta\left[\left(x^{\nu}-x^{\prime \nu}\right)^{2}\right] \\
& D_{a \nu}(\zeta)=\frac{1}{2 \pi} \theta\left(x^{\prime 0}-x^{0}\right) \delta\left[\left(x^{\nu}-x^{\prime \nu}\right)^{2}\right] .
\end{aligned}
$$

As relações 70 e (71) determinam as funções de Green retardada e avançada, respectivamente, como expressões invariantes. Vale mencionar que a função teta, aparentemente não-invariante, quando submetida às restrições da função delta, torna-se invariante sob transformações de Lorentz próprias.

As funções teta e delta em 70 mostram que a função de Green avançada contribui unicamente na frente do cone de luz do ponto de observação, enquanto que na expressão (71), essas funções indicam que a função de Green retardada contribui apenas atrás desse cone de luz (figura 5).

Com efeito, podemos reescrever a solução da equação de onda 25 em termos das funções de Green, isto é,

$$
A^{\alpha}(x)=A_{i n}^{\alpha}(x)+\int D_{r e t}\left(x-x^{\prime}\right) J^{\alpha}(x) d^{4} x^{\prime}
$$




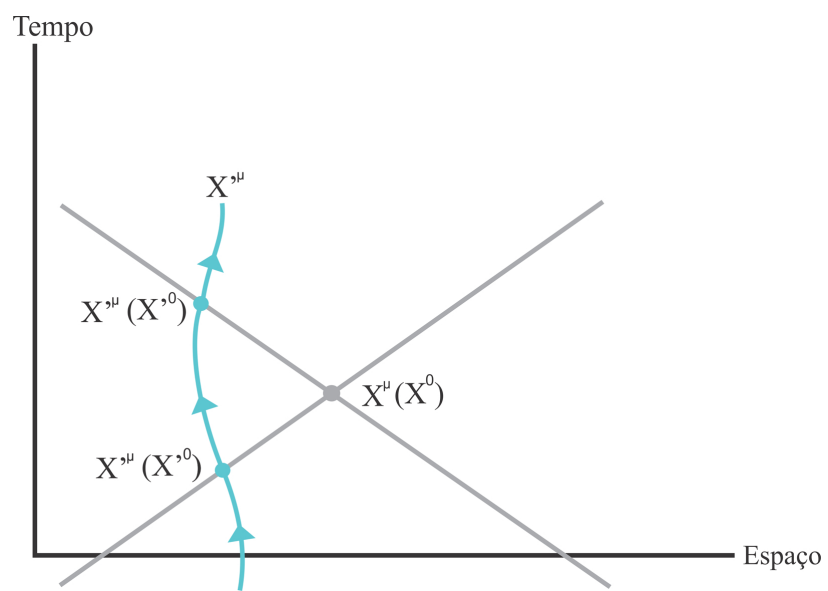

Figura 5: Pontos de intersecção entre a linha de mundo da fonte e o cone de luz passado e futuro do ponto de observação.

ou

$$
A^{\alpha}(x)=A_{e m}^{\alpha}(x)+\int D_{a \nu}\left(x-x^{\prime}\right) J^{\alpha}\left(x^{\prime}\right) d^{4} x^{\prime}
$$

onde os termos $A_{i n}^{\alpha}$ e $A_{e m}^{\alpha}$ correspondem respectivamente aos quadripotenciais incidente e emergente, sendo ambos soluções da equação de onda homogênea. É conveniente verificar que se admitirmos $\left(x^{0}-x^{\prime}\right) \rightarrow+\infty$ nas equações 72 e 73 , as integrais das fontes se anulam em razão da natureza das funções de Green retardada e avançada. Nesse caso, estamos diante do potencial de campo livre incidente $A_{i n}^{\alpha}(x)$ e potencial de campo livre emergente $A_{e m}^{\alpha}(x)$, respectivamente.

Para compreendermos adequadamente o quadrivetor $J^{\mu}(x)$, de início, consideremos que a fonte seja um carga elétrica cuja posição em um dado referencial inercial é $\vec{r}(t)$ e que as densidades de carga e corrente, na devida ordem, sejam dadas pelas expressões

$$
\begin{gathered}
\rho(\vec{x}, t)=e \delta[\vec{x}-\vec{r}(t)] \\
\vec{J}(\vec{x}, t)=e \vec{v}(t) \delta[\vec{x}-\vec{r}(t)]
\end{gathered}
$$

onde $\vec{v}=\frac{d \vec{r}}{d t}$ representa a velocidade da carga no referencial dado.

As densidades de carga e de corrente também podem ser representadas como componentes de um tensor de primeira ordem, ou quadrivetor, da seguinte forma 32

$$
J^{\alpha}(x)=e \int U^{\alpha}(\tau) \delta^{(4)}\left[x^{\mu}-r^{\mu}(\tau)\right] d \tau,
$$

na qual, $r^{\alpha}(\tau)=[t, \vec{r}(t)]$ e $U^{\alpha}(\tau)=[\gamma, \gamma \vec{v}]$ correspondem, nesta ordem, à posição e velocidade no espaçotempo de Minkowski. Acentua-se que a quadricorrente $J^{\alpha}(x)$ é definida em termos de componentes como $J^{\alpha}(x)=$ $(\rho, \vec{J})$.

Calculando explicitamente as componentes espaciais e temporal de $J^{\alpha}(x)$, obtemos novamente as relações (74) e (75).

$$
\begin{gathered}
\rho(\vec{x}, t)=J^{0}(x)=e \int U^{0}(\tau) \delta(t-\tau) \delta^{(4)}[\vec{x}-\vec{r}(\tau)] d \tau \\
\rho(\vec{x}, t)=J^{0}(x)=e \delta[\vec{x}-\vec{r}(t)] \\
J(x)=e \int \gamma \vec{v}(\tau) \delta(t-\tau) \delta[\vec{x}-\vec{r}(t)] d \tau \\
\vec{J}(\vec{x}, t)=e \vec{v}(t) \delta[\vec{x}-\vec{r}(t)]
\end{gathered}
$$

\section{Obtenção do propagador de Feynman}

Em particular, há uma importante função de Green invariante denominada de propagador de Feynman. Para esse propagador 32 definido por

$D_{F}\left(x-x^{\prime}\right)=\theta\left(\zeta^{0}\right) D^{+}\left(x-x^{\prime}\right)+\theta\left(-\zeta^{0}\right) D^{-}\left(x-x^{\prime}\right)$ (77) dependendo do sinal de $\left(x^{0}-x^{\prime} 0\right)$ obtemos a contribuição de um ou outro polo separadamente, sendo estas contribuições representadas pelas funções $D^{+}$e $D^{-}$. Enfatizase que $D^{+}$e $D^{-}$não são funções de Green quando analisadas isoladamente haja vista que não satisfazem a expressão 26.).

Para obtermos o propagador de Feynman como uma combinação das funções $D^{+}$e $D^{-}$, inicialmente, obteremos as integrais de resíduos destas funções.

De início, determinaremos $D^{-}$tomando o contorno fechado $F_{1}$ e deslocando no eixo imaginário o polo $k^{0}=$ $-|\vec{k}|$ de $+\varepsilon$ e polo $k^{0}=|\vec{k}|$ de $-\varepsilon$ e resolvendo a integral (36) para este contorno (figura 6). Procedendo deste modo, teremos

$$
\int_{F_{1}} f\left(k^{0}\right) d k^{0}=-2 \pi i \sum r e s,
$$

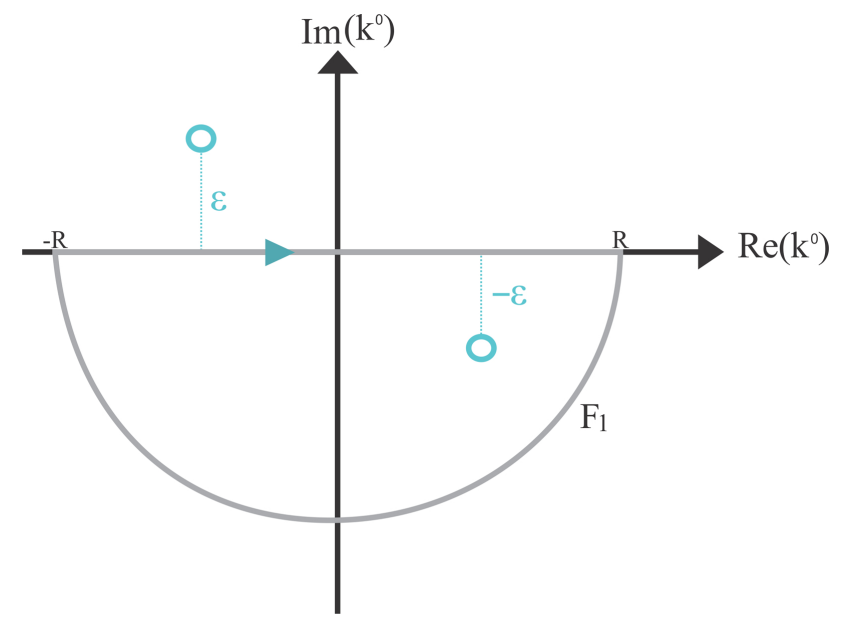

Figura 6: Os polos deslocados no eixo imaginário da quantidade $+\epsilon$ para $k^{0}=-|\vec{k}|$ e da quantidade $-\epsilon$ para $k^{0}=+|\vec{k}|$ onde $\circ$ contorno fechado $F_{1}$ contém o polo deslocado $k^{0}=+|\vec{k}|-i \epsilon$. 
onde $f\left(k^{0}\right)=\left\{\left[\left(k^{0}\right)^{2}-|\vec{k}|^{2}\right]^{-1} e^{-i k^{0} \zeta^{0}}\right\}$.

Atente que nesse caso:

$$
\int_{F_{1}} f\left(k^{0}\right) d k^{0}=\int_{-R}^{+R} f\left(k^{0}\right) d k^{0}+\int_{C_{1}} f\left(k^{0}\right) d k^{0} .
$$

Conforme foi analisado anteriormente, admitindo $R \rightarrow$ $\infty$ e que $\lim _{R \rightarrow \infty}\left[\int_{C_{1}} f\left(k^{0}\right) d k^{0}\right]=0$, então:

$$
\int_{-\infty}^{+\infty} f\left(k^{0}\right) d k^{0}=-2 \pi i \sum r e s .
$$

Como o contorno fechado $F_{1}$ contém apenas o polo $k^{0}=|\vec{k}|-i \varepsilon$, alcançamos

$$
\begin{aligned}
& \sum r e s=\lim _{k^{0} \rightarrow|\vec{k}|-i \varepsilon}\left\{\left[\frac{k^{0}-(|\vec{k}|-i \varepsilon)}{k^{0}-(|\vec{k}|-i \varepsilon)}\right]\right. \\
&\left.\times\left[\frac{e^{\left(-i k^{0} \zeta^{0}\right)}}{k^{0}-(-|\vec{k}|-i \varepsilon)}\right]\right\}
\end{aligned}
$$

e, deste modo, a expressão 80, pode ser reescrita como:

$$
\int_{-\infty}^{+\infty} f\left(k^{0}\right) d k^{0}=-2 \pi i\left[\frac{e^{-(|\vec{k}|-i \varepsilon) \zeta^{0}}}{(2|\vec{k}|-2 i \varepsilon)}\right] .
$$

Por fim, admitindo $\varepsilon \rightarrow 0$, obtemos:

$$
\lim _{\varepsilon \rightarrow 0}\left[\int_{-\infty}^{+\infty} f\left(k^{0}\right) d k^{0}\right]=-\pi i \frac{e^{-i|\vec{k}| \zeta^{0}}}{|\vec{k}|} .
$$

De posse dessas relações, podemos definir previamente $D^{+}\left(x-x^{\prime}\right)$ pela seguinte expressão:

$$
D^{+}\left(x-x^{\prime}\right)=-\int \frac{e^{i \vec{k} \vec{R}}}{16 \pi^{4}} d^{3} \vec{k} \int\left[\frac{e^{-i k^{0} \zeta^{0}}}{\left(k^{0}\right)^{2}-|\vec{k}|^{2}}\right] d k^{0} .
$$

Considerando o polo contido no contorno fechado $F_{1}$ (figura 6) e usando o resultado da integral de resíduo (82), a equação 83 resultará em:

$$
D^{+}\left(x-x^{\prime}\right)=\frac{i}{16 \pi^{3}|\vec{k}|} \int e^{i \vec{k} \vec{R}} e^{-i|\vec{k}| \zeta^{0}} d^{3} \vec{k}
$$

Sabendo que $\int \delta\left(k^{0}-|\vec{k}|\right) e^{-i k^{0} \zeta^{0}} d k^{0}=e^{-i|\vec{k}| \zeta^{0}} \mathrm{e}$ após algumas manipulações algébricas, obtemos a expressão,

$$
\begin{aligned}
D^{+}\left(x-x^{\prime}\right)= & \frac{i}{|\vec{k}|} \int \frac{e^{i \vec{k} \vec{R}}}{16 \pi^{3}} d^{3} \vec{k}\left[\int \delta\left(k^{0}-|\vec{k}|\right) e^{-i k^{0} \zeta^{0}} d k^{0}\right] \theta\left(k^{0}\right) \\
& +\frac{i}{|\vec{k}|} \int \frac{e^{i \vec{k} \vec{R}}}{16 \pi^{3}} d^{3} \vec{k}\left[\int \delta\left(k^{0}+|\vec{k}|\right) e^{-i k^{0} \zeta^{0}} d k^{0}\right] \theta\left(k^{0}\right)
\end{aligned}
$$

onde

$$
\theta\left(k^{0}\right)=\left\{\begin{array}{lll}
1, & \text { se } \quad k^{0}>0 \\
0, & \text { se } \quad k^{0}<0
\end{array}\right.
$$

Considerando a relação

$$
\delta\left(k^{\mu} k_{\mu}\right)=\frac{\delta\left(k^{0}-|\vec{k}|\right)+\delta\left(k^{0}+|\vec{k}|\right)}{2|\vec{k}|}
$$

e alocando-a na equação (85), alcançamos:

$$
D^{+}\left(x-x^{\prime}\right)=\frac{i}{8 \pi^{3}} \int e^{-i k^{\mu} \zeta_{\mu}} \delta\left(k^{\mu} k_{\mu}\right) \theta\left(k^{0}\right) d^{4} k
$$

Para determinarmos a função $D^{-}$, o procedimento é similar ao que foi realizado para obtermos a função $D^{+}$, porém, nesse caso, tomamos o contorno fechado $F_{2}$ e promovemos o deslocamento no eixo imaginário o polo $k^{0}=-|\vec{k}|$ de $\varepsilon$ e o polo $k^{0}=|\vec{k}|$ de $-\varepsilon$, conforme ilustra a figura 7 .

Deste modo, teremos

$$
\int_{F_{2}} f\left(k^{0}\right) d k^{0}=2 \pi i \sum r e s,
$$

onde

$$
\int_{F_{2}} f\left(k^{0}\right) d k^{0}=\int_{-R}^{+R} f\left(k^{0}\right) d k^{0}+\int_{C_{2}} f\left(k^{0}\right) d k^{0} .
$$

Tomando o limite em que $R \rightarrow \infty$ e que para tal condição a integral em $C_{2}$ é nula, a equação (88) resulta em:

$$
\int_{-\infty}^{+\infty} f\left(k^{0}\right) d k^{0}=2 \pi i \sum r e s
$$


Em razão do fato de o contorno fechado $F_{2}$ conter apenas o polo $k^{0}=|\vec{k}|+i \varepsilon$, o somatório dos resíduos pode ser descrito por

$$
\lim _{\varepsilon \rightarrow 0}\left[\int_{-\infty}^{+\infty} f\left(k^{0}\right) d k^{0}\right]=-\pi i \frac{e^{i|\vec{k}| \zeta^{0}}}{|\vec{k}|} .
$$

e, por conseguinte:

$\sum r e s=\lim _{k^{0} \rightarrow-|\vec{k}|+i \varepsilon}\left[\frac{k^{0}-(i \varepsilon-|\vec{k}|)}{k^{0}-(|\vec{k}|-i \varepsilon)}\right]\left[\frac{e^{\left(-i k^{0} \zeta^{0}\right)}}{k^{0}-(i \varepsilon-|\vec{k}|)}\right]$

e, desta forma, substituindo esse último resultado na expressão 89, teremos

$$
\int_{-\infty}^{+\infty} f\left(k^{0}\right) d k^{0}=2 \pi i\left[\frac{e^{-i(-|\vec{k}|+i \varepsilon) \zeta^{0}}}{(-2|\vec{k}|+2 i \varepsilon)}\right]
$$

Empregando novamente a condição tal que $\varepsilon \rightarrow 0$, temos

$$
\begin{aligned}
D^{-}\left(x-x^{\prime}\right) & =\frac{i}{|\vec{k}|} \int \frac{e^{i \vec{k} \vec{R}}}{16 \pi^{3}} d^{3} \vec{k}\left[\int \delta\left(k^{0}+|\vec{k}|\right) e^{-i k^{0} \zeta^{0}} d k^{0}\right] \theta\left(-k^{0}\right) \\
& +\frac{i}{|\vec{k}|} \int \frac{e^{i \vec{k} \vec{R}}}{16 \pi^{3}} d^{3} \vec{k}\left[\int \delta\left(k^{0}-|\vec{k}|\right) e^{-i k^{0} \zeta^{0}} d k^{0}\right] \theta\left(-k^{0}\right)
\end{aligned}
$$

onde

$$
\theta\left(-k^{0}\right)= \begin{cases}1, & \text { se }-k^{0}>0 \\ 0, & \text { se }-k^{0}<0\end{cases}
$$

Aplicando a relação 86, podemos reescrever (94) como:

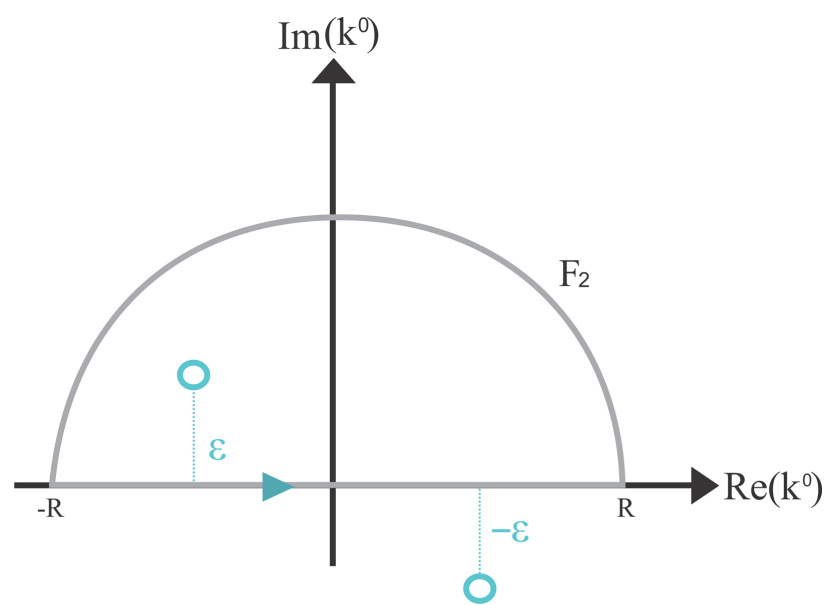

Figura 7: Os polos deslocados no eixo imaginário da quantidade $+\epsilon$ para $k^{0}=-|\vec{k}|$ e da quantidade $-\epsilon$ para $k^{0}=+|\vec{k}|$ onde $\circ$ contorno fechado $F_{2}$ contém o polo deslocado $k^{0}=-|\vec{k}|+i \epsilon$.
(92)

Avaliando o polo contido no contorno $F_{2}$ e usando o resultado da integral dada em (91), a expressão 92 torna-se:

$$
D^{-}\left(x-x^{\prime}\right)=\frac{i}{16 \pi^{3}|\vec{k}|} \int e^{i \vec{k} \vec{R}} e^{i|\vec{k}| \zeta^{0}} d^{3} \vec{k}
$$

Sabendo que $\int \delta\left(k^{0}+|\vec{k}|\right) e^{-i k^{0} \zeta^{0}} d k^{0}=e^{i|\vec{k}| \zeta^{0}}$ e desenvolvendo a equação 93 , obtemos 
quentemente nos permite escrever o potencial produzido por esta carga em movimento como:

$$
A^{\mu}(x)=\int D_{r e t}\left(x-x^{\prime}\right) J^{\mu}\left(x^{\prime}\right) d^{4} x^{\prime}
$$

Substituindo adequadamente a equação 76 na equação (97) e usando a relação 70 , podemos reescrever a equação 97 e obtermos

$$
A^{\mu}(x)=\frac{e}{2 \pi} \int d^{4} x^{\prime} d \tau U^{\mu}(\tau) \delta^{(4)}\left[x^{\prime}-r(\tau)\right] \Lambda
$$

onde $\Lambda=\delta\left[\left(x-x^{\prime}\right)^{2}\right] \theta\left(x^{0}-x^{0^{\prime}}\right)$

De fato, para resolvermos a integral em $d^{4} x^{\prime}$ empregaremos a propriedade da filtragem do delta de Dirac. A aplicação dessa filtragem está expressa do seguinte modo:

$$
\begin{aligned}
A^{\mu}(x) & =\frac{e}{2 \pi} \int_{-\infty}^{+\infty} U^{\mu}(\tau) \delta\left[\left(x^{\mu}-r^{\mu}(\tau)\right)^{2}\right] \\
& \times \theta\left[x^{0}-r^{0}(\tau)\right] d \tau
\end{aligned}
$$

É relevante atentar para o fato de que a integral sobre o tempo próprio da carga contribui unicamente em $\tau=\tau_{0}$, onde $\tau_{0}$ é definido pela condição do cone de luz, conforme ilustra a figura 8 .

Aplicando a propriedade do delta dada por

$$
\delta[f(\tau)]=\sum_{i} \delta\left(\tau-\tau_{i}\right)\left|\left(\frac{d f}{d \tau}\right)_{\tau=\tau_{i}}\right|^{-1}
$$

onde $f(\tau)=[x-r(\tau)]^{\mu}[x-r(\tau)]_{\mu}$ e a diferenciando, teremos a relação

$$
\frac{d f}{d \tau}=-2 U_{\alpha}(x-r)^{\alpha}
$$

na qual, $U_{\mu}(\tau)=\gamma(1, \vec{v})$.

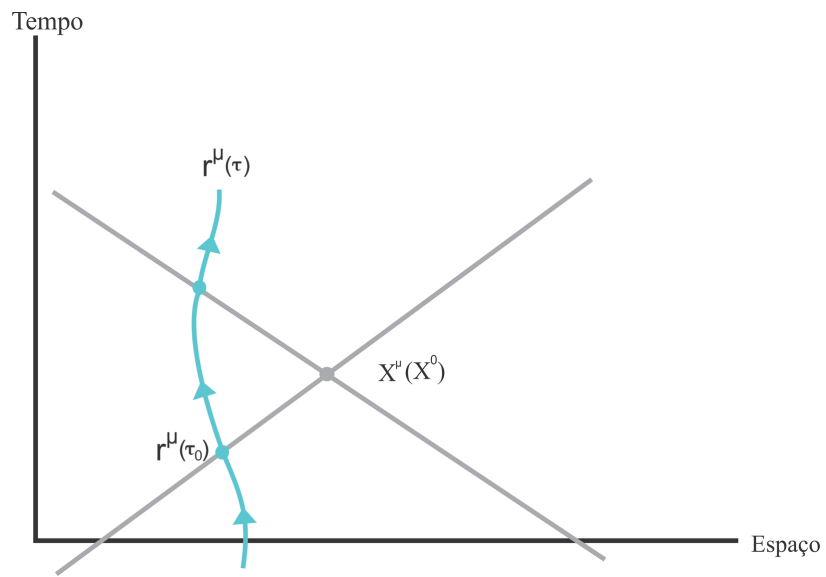

Figura 8: $O$ ponto de intersecção entre a linha de mundo da fonte e o cone de luz passado do ponto de observação.
Desenvolvendo a expressão 100 , temos:

$$
\delta[f(\tau)]=\frac{\delta\left(\tau-\tau_{p}\right)+\delta\left(\tau-\tau_{f}\right)}{2\left|U_{\mu}(x-r)^{\mu}\right|} .
$$

Nesse ínterim, considerando $\vec{x}-\vec{r}\left(\tau_{0}\right)=\vec{R}$, temos pelo cone de luz que $x^{0}-r^{0}\left(\tau_{0}\right)=R$. Ademais, definindo as relações $\vec{R}=R \hat{R}, \vec{\beta}=\vec{v}$ e $\gamma^{-2}=1-\beta^{2}$, podemos reescrever o produto escalar $U_{\mu}(x-\mu)^{\mu}$ como:

$$
U_{\mu}(x-r)^{\mu}=\eta_{\nu \mu} U^{\nu}(x-r)^{\mu}=\gamma R(1-|\vec{\beta}|) .
$$

Uma vez que a expressão 103 é positiva, pois $|\vec{\beta}|<1$, podemos reescrever a equação 102 sem a relação de módulo, isto é:

$$
\delta[f(\tau)]=\frac{\delta\left(\tau-\tau_{p}\right)+\delta\left(\tau-\tau_{f}\right)}{2 U_{\mu}(x-r)^{\mu}} .
$$

Substituindo as expressões 103 e 104 na equação 99, temos:

$$
\begin{aligned}
A^{\mu}(x)= & \frac{e}{4 \pi}\left\{\frac{U^{\mu}\left(\tau_{p}\right) \theta\left[x^{0}-r^{0}\left(\tau_{p}\right)\right]}{U_{\alpha}\left(\tau_{p}\right)\left[x-r\left(\tau_{p}\right)\right]^{\alpha}}\right\}+ \\
& \frac{e}{4 \pi}\left\{\frac{U^{\mu}\left(\tau_{f}\right) \theta\left[x^{0}-r^{0}\left(\tau_{f}\right)\right]}{U_{\alpha}\left(\tau_{f}\right)\left[x-r\left(\tau_{f}\right)\right]^{\alpha}}\right\}
\end{aligned}
$$

Admitindo a condição do cone de luz passado, $x^{0}>$ $r^{0}\left(\tau_{p}\right)$, e definido $\tau_{p}=\tau_{0}$, podemos exprimir a equação 105 como:

$$
A^{\mu}(x)=\frac{e}{4 \pi}\left\{\frac{U^{\mu}(\tau)}{U_{\alpha}(\tau)[x-r(\tau)]^{\alpha}}\right\}_{\tau=\tau_{0}} .
$$

Decompondo o quadrivetor $A^{\mu}(x)$ nas suas componentes temporal e espacial, obtemos respectivamente as seguintes expressões

$$
\begin{aligned}
& A^{0}(x)=\psi(\vec{x}, t)=\frac{e}{4 \pi}\left[\frac{1}{(1-\vec{\beta} \hat{R}) R}\right]_{r e t} \\
& A^{i}(x)=\vec{A}(\vec{x}, t)=\frac{e}{4 \pi}\left[\frac{\vec{\beta}}{(1-\vec{\beta} \hat{R}) R}\right]_{r e t}
\end{aligned}
$$

denominadas de potenciais retardados de Liénard-Wiechert.

Nesse contexto, uma outra etapa fundamental consiste na obtenção do tensor do campo eletromagnético. Para tal propósito, determinemos inicialmente $\partial^{\nu} A^{\mu}$. Realizando a substituição da equação 76 na equação 97 e integrando em $d^{4} x^{\prime}$, teremos:

$$
A^{\mu}(x)=e \int_{-\infty}^{+\infty} U^{\mu}(\tau) D_{r e t}[x-r(\tau)] d \tau .
$$


Derivando essa última expressão em relação às coordenadas $x^{\nu}$, obtemos

$$
\partial^{\nu} A^{\mu}=e \int_{-\infty}^{+\infty} U^{\mu} \frac{\partial D_{r}}{\partial\left[(x-r)^{2}\right]} \frac{\partial\left[(x-r)^{2}\right]}{\partial x_{\nu}} d \tau
$$

que, por sua vez, pode ser transformada em

$$
\partial^{\nu} A^{\mu}=2 e \int_{-\infty}^{+\infty} d \tau[x-r(\tau)]^{\nu} U^{\mu} \frac{d D_{r}}{d \tau} \frac{d \tau}{d\left[(x-r(\tau))^{2}\right]}
$$

$$
\text { diante do fato de que } \frac{\partial\left[(x-r)^{\alpha}(x-r)_{\alpha}\right]}{\partial x_{\nu}}=2(x-r)^{\nu} \text {. }
$$

De fato, podemos ainda simplificar a equação (111) ao fazermos uso das equações (100) e 101), o que nos retorna a expressão

$$
\partial^{\nu} A^{\mu}=-e \int_{-\infty}^{+\infty} d \tau \frac{(x-r)^{\nu} U^{\mu}}{(x-r)^{\alpha} U_{\alpha}} \frac{d D_{r}}{d \tau}
$$

Integrando por partes a expressão 112 e assumindo que para instantes diferentes de $\tau_{0}$,

$$
\delta\left[(x-r(\tau))^{2}\right]=0
$$

além do fato de que a radiação da carga pontual emitida num instante infinito (ou também emitida de uma distância infinita do ponto de observação) não será efetivamente detectada, então, alcançamos a expressão:

$$
\partial^{\nu} A^{\mu}=\frac{e}{2 \pi} \int_{-\infty}^{+\infty} d \tau \theta\left(x^{0}-r^{0}(\tau)\right) \delta\left[(x-r(\tau))^{2}\right] \frac{d}{d t}\left[\frac{(x-r)^{\nu} U^{\mu}}{(x-r)^{\alpha} U_{\alpha}}\right]
$$

Fazendo uso da relação (104) nessa última equação, chegaremos na expressão (114), na qual, usamos o delta de Dirac para efetuar a integral em $d \tau$.

$$
\begin{aligned}
\partial^{\nu} A^{\mu}= & \frac{\theta\left[x^{0}-r^{0}\left(\tau_{p}\right)\right] e(4 \pi)^{-1}}{\left[x-r\left(\tau_{p}\right)\right]^{\alpha} U_{\alpha}\left(\tau_{p}\right)} \frac{d}{d \tau}\left[\frac{\left(x-r\left(\tau_{p}\right)\right)^{\nu} U^{\mu}\left(\tau_{p}\right)}{\left(x-r\left(\tau_{p}\right)\right)^{\alpha} U_{\alpha}\left(\tau_{p}\right)}\right]+ \\
& \frac{\theta\left[x^{0}-r^{0}\left(\tau_{f}\right)\right] e(4 \pi)^{-1}}{\left[x-r\left(\tau_{f}\right)\right]^{\alpha} U_{\alpha}\left(\tau_{f}\right)} \frac{d}{d \tau}\left[\frac{\left(x-r\left(\tau_{f}\right)\right)^{\nu} U^{\mu}\left(\tau_{f}\right)}{\left(x-r\left(\tau_{f}\right)\right)^{\alpha} U_{\alpha}\left(\tau_{f}\right)}\right]
\end{aligned}
$$

Considerando a condição do cone de luz passado, $x^{0}>$ $r^{0}\left(\tau_{0}\right)$, a expressão 114 torna-se

$$
\partial^{\nu} A^{\mu}=\frac{e\left[4 \pi U_{\alpha}\left(\tau_{0}\right)\right]^{-1}}{\left[x-r\left(\tau_{0}\right)\right]^{\alpha}} \frac{d}{d \tau}\left[\frac{\left(x-r\left(\tau_{0}\right)\right)^{\nu} U^{\mu}\left(\tau_{0}\right)}{\left(x-r\left(\tau_{0}\right)\right)^{\alpha} U_{\alpha}\left(\tau_{0}\right)}\right]
$$

que também pode reescrita como

$$
\partial^{\nu} A^{\mu}=\frac{e\left[4 \pi U_{\alpha}\left(\tau_{0}\right)\right]^{-1}}{\left[x-r\left(\tau_{0}\right)\right]^{\alpha}} \frac{d}{d \tau}\left[\frac{\left(x-r\left(\tau_{0}\right)\right)^{\mu} U^{\nu}\left(\tau_{0}\right)}{\left(x-r\left(\tau_{0}\right)\right)^{\alpha} U_{\alpha}\left(\tau_{0}\right)}\right]
$$

Substituindo essas últimas relações na equação 18 , obtemos:

$$
F^{\mu \nu}=\frac{e(4 \pi)^{-1}}{(x-r)^{\alpha} U_{\alpha}} \frac{d}{d \tau}\left[\frac{(x-r)^{\mu} U^{\nu}-(x-r)^{\nu} U^{\mu}}{(x-r)^{\alpha} U_{\alpha}}\right] .
$$

Aplicando a regra da derivada do produto nessa última expressão, chegamos na seguinte relação

$$
\begin{aligned}
& F^{\mu \nu}=\frac{e(4 \pi)^{-1}}{\left[(x-r)^{\alpha} U_{\alpha}\right]^{3}}\left[(x-r)^{\mu} \alpha^{\nu}-(x-r)^{\nu} \alpha^{\mu}\right] \\
& \times\left[(x-r)^{\alpha} U_{\alpha}\right]-\frac{e(4 \pi)^{-1}}{\left[(x-r)^{\alpha} U_{\alpha}\right]^{3}}\left[(x-r)^{\mu} U^{\nu}\right. \\
& \left.-(x-r)^{\nu} U^{\mu}\right] \frac{d}{d \tau}\left[(x-r)^{\alpha} U_{\alpha}\right]
\end{aligned}
$$

na qual, $a^{\nu}=\left[\gamma^{4} \vec{B} \vec{a}, \gamma^{2} \vec{a}+\gamma^{4} \vec{\beta}(\vec{\beta} \vec{a})\right]$ consiste na quadriaceleração.

Sabendo que campos elétrico e magnético são definidos em termos dos potenciais escalares $\psi$ e/ou do vetor $\vec{A}$, isto é

$$
\begin{gathered}
\vec{E}(\vec{x}, t)=-\vec{\nabla} \psi-\frac{\partial \vec{A}}{\partial t} \\
\vec{B}(\vec{x}, t)=\vec{\nabla} \times \vec{A}
\end{gathered}
$$

e lembrando que $A^{\mu}=(\psi, \vec{A})$ e $F^{\mu \nu}=\partial^{\mu} A^{\nu}-\partial^{\nu} A^{\mu}$, verifica-se facilmente as seguintes relações:

$$
\begin{gathered}
F^{o o}=F^{i i}=0, \\
F^{o i}=-F^{i o}=-E^{i} .
\end{gathered}
$$


Nesse caso, $E^{1}=E^{x}, E^{2}=E^{y}$ e $E^{3}=E^{z}$, além de $F^{12}=-B^{z}, F^{13}=B^{y}$ e $F^{23}=-B^{x}$. Efetuando as manipulações algébricas adequadas com a expressão (116), encontramos a seguinte expressão do vetor do campo elétrico:

$$
\begin{aligned}
\vec{E}= & \frac{e}{4 \pi}\left[\frac{(\hat{R}-\vec{B})}{(\gamma R)^{2}(1-\vec{\beta} \hat{R})^{3}}\right]_{r e t}+ \\
& \frac{e}{4 \pi}\left\{\frac{[\hat{R} \times((\hat{R}-\vec{\beta}) \times \vec{\alpha})]}{(1-\vec{\beta} \hat{R})^{3} R}\right\}_{\text {ret }} .
\end{aligned}
$$

Ademais, fazendo uso das componentes puramente espaciais do tensor de ordem dois $F^{\mu \nu}$, podemos alcançar a expressão vetorial do campo magnético dada por:

$$
\begin{aligned}
\vec{B}=\hat{R} \times \vec{E}= & \frac{e}{4 \pi}\left[\frac{\hat{R} \times(\hat{R}-\vec{B})}{\left.(\gamma R)^{2}(1-\vec{\beta} \hat{R})^{3}\right]_{\text {ret }}}+\right. \\
& \frac{e}{4 \pi}\left\{\frac{\hat{R} \times[\hat{R} \times((\hat{R}-\vec{\beta}) \times \vec{\alpha})]}{(1-\vec{\beta} \hat{R})^{3} R}\right\}_{\text {ret }} .
\end{aligned}
$$

Empregando a definição $\vec{S}=[\vec{E} \times \vec{B}]$ para o vetor de Poynting e integrando em uma superfície fechada com raio infinitamente grande $\oiint \vec{S} d \vec{A}$, verifica-se a predominância dos termos que decaem com $R^{-1}$ em comparação àqueles que decaem com $R^{-2}$. Em detalhe, os termos predominantes são chamados de campo elétrico de radiação $\left(\vec{E}_{r a d}\right)$ e campo magnético de radiação $\left(\vec{B}_{\text {rad }}\right)$, sendo descritos respectivamente pelas relações abaixo.

$$
\begin{gathered}
\vec{E}_{\text {rad }} \cong \frac{e}{4 \pi}\left\{\frac{[\hat{R} \times((\hat{R}-\vec{\beta}) \times \vec{\alpha})]}{(1-\vec{\beta} \hat{R})^{3} R}\right\}_{r e t} \\
\vec{B}_{\text {rad }} \cong \frac{e}{4 \pi}\left\{\frac{\hat{R} \times[\hat{R} \times((\hat{R}-\vec{\beta}) \times \vec{\alpha})]}{(1-\vec{\beta} \hat{R})^{3} R}\right\}_{r e t}
\end{gathered}
$$

Observando as expressões dos campos elétrico e magnético de radiação é relevante atentar para o fato de que tais campos dependem explicitamente da aceleração da partícula carregada, evidenciando assim que cargas pontuais aceleradas, em relação a um certo sistema de coordenadas inercial, emitem radiação eletromagnética!

Admitindo o cálculo desses campos a partir de um referencial instantaneamente em repouso com a carga acelerada, onde $\vec{v}=0$ e, por consequência, $\vec{\beta}=0$, as expressões dos campos assumem formas mais simples. Nesse caso, o campo elétrico se reduz à equação

$$
\vec{E}_{\text {rad }} \cong \frac{e}{4 \pi}\left[\frac{\hat{R} \times(\hat{R} \times \vec{a})}{R}\right]_{\text {ret }}
$$

cujo módulo é dado pela expressão

$$
\left|\vec{E}_{r a d}\right| \cong \frac{e}{4 \pi} \frac{a \operatorname{sen}(\theta)}{R}
$$

enquanto que o campo magnético, por sua vez, pode ser reduzido à equação

$$
\vec{B}_{r a d} \cong \frac{e}{4 \pi}\left\{\frac{\hat{R} \times[\hat{R} \times(\hat{R} \times \vec{a})]}{R}\right\}_{r e t}
$$

sendo o módulo desse campo representado por:

$$
\left|\vec{B}_{\text {rad }}\right| \cong \frac{e}{4 \pi} \frac{a \operatorname{sen}(\theta)}{R} .
$$

Em ambos os casos (equações $122 \mathrm{e} 124$, $\theta$ indica o ângulo entre a aceleração e a direção do ponto de observação.

Para obtermos o vetor de Poynting é necessário abordarmos o tensor energia-momento canônico que no caso do campo eletromagnético é definido por:

$$
T^{\mu \nu}=F^{\mu \rho} F_{\rho}^{\nu}+\frac{1}{4} g^{\mu \nu} F^{\rho \sigma} F_{\rho \sigma} .
$$

Sabendo que as componentes $T^{0 i}$ deste tensor estão diretamente relacionadas com as componentes $S^{i}$ do vetor de Poynting $\vec{S}$, teremos

$$
T^{0 i}=F^{0 \rho} F_{\rho}^{i}=S^{i} .
$$

Desse modo, o fluxo instantâneo de energia por unidade de área e tempo (vetor de Poynting) será dado por

$$
\vec{S}=\left[\vec{E}_{r a d} \times \vec{B}_{r a d}\right]
$$

onde são considerados apenas os campos que contribuem com o fluxo de energia a longas distâncias.

Uma vez que os campos $\vec{E}$ e $\vec{B}$ são perpendiculares, temos que $\vec{B}=R \times \vec{E}$, o que nos permite reescrever a equação 125 como:

$$
\begin{array}{r}
\vec{S}=\left|\vec{E}_{\text {rad }}\right|\left|\vec{B}_{\text {rad }}\right| \operatorname{sen}\left(\frac{\pi}{2}\right) \hat{R}=\left|\vec{E}_{\text {rad }}\right|^{2} \hat{R} \\
\vec{S}=\left[\frac{e|\vec{a}| \operatorname{sen}(\theta)}{4 \pi R}\right]^{2} \hat{R}=|\vec{S}| \hat{R} .
\end{array}
$$

A figura 9 ilustra o comportamento da radiação de uma carga movendo-se com baixa velocidade. O padrão de intensidade da radiação tem um formato toroidal com a máxima emissão situada no plano equatorial.

Sendo a potência irradiada por unidade de ângulo sólido $d \Omega$ dada por 


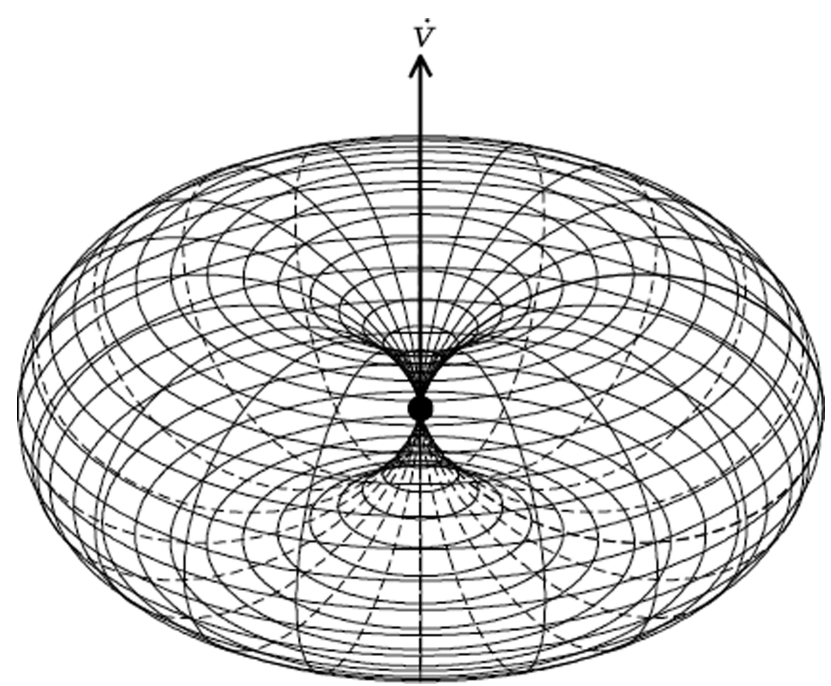

Figura 9: Padrão de radiação emitida por uma carga em condição não-relativística, na qual, a máxima emissão ocorre no plano perpendicular à aceleração da partícula [33].

$$
\frac{d P}{d \Omega}=\vec{S} R^{2}
$$

onde, nesse caso, $d \Omega=\operatorname{sen}(\theta) d \theta d \phi$, a potência total emitida pela carga é obtida integrando a equação (127), ou seja:

$$
P=\oiint \frac{d P}{d \Omega} d \Omega
$$

Substituindo adequadamente as relações 126 e 127 na expressão 128, alcançamos finalmente

$$
\begin{gathered}
P=\left(\frac{e|\vec{a}|}{4 \pi}\right)^{2} \oiint[\operatorname{sen}(\theta)]^{3} d \theta d \phi \Longrightarrow \\
P=\frac{e^{2}|\vec{a}|^{2}}{6 \pi}
\end{gathered}
$$

que consiste na potência total irradiada por uma carga elétrica acelerada, denominada de potência ou fórmula de Larmor 34, 35].

A potência de Larmor 129 também pode ser disposta em um forma covariante, utilizando para isso, sobretudo, argumentos de invariância sob transformações de Lorentz próprias. Para encontrarmos a generalização apropriada, reescrevemos a potência de Larmor na forma

$$
P=\frac{e^{2}}{6 \pi m^{2}}\left(\frac{d \vec{p}}{d t} \cdot \frac{d \vec{p}}{d t}\right),
$$

onde $m$ corresponde a massa da partícula carregada e $\vec{p}$ o seu momento linear. A generalização covariante de Lorentz é, portanto, dada por

$$
P=-\frac{e^{2}}{6 \pi m^{2}}\left(\frac{d p_{\mu}}{d \tau} \frac{d p^{\mu}}{d \tau}\right),
$$

na qual, $d \tau=\gamma^{-1} d t$ é o elemento de tempo próprio e $p^{\mu}=(E, m \vec{v})$ é o quadrivetor momento-energia da partícula carregada. Perspicazmente, se admitirmos um referencial instantaneamente em repouso com a carga acelerada, em que $\vec{v}=0$ e $\vec{\beta}=0$, a expressão 130 se reduz à expressão 129 .

\section{Fórmula ou generalização de Liénard}

Para encontrarmos uma expressão geral para a potência total irradiada por uma carga acelerada, consideremos $E=\gamma m$ e $\vec{p}=E \vec{v}$. Nesse sentido, podemos reescrever a equação 130 como:

$$
P=-\frac{e^{2}}{6 \pi m^{2}}\left(g_{\mu \nu} \frac{d p^{\mu}}{d \tau} \frac{d p^{\mu}}{d \tau}\right),
$$

Efetuando as devidas manipulações algébricas como se segue

$$
\begin{gathered}
P=-\frac{e^{2}}{6 \pi m^{2}}\left(g_{00} \frac{d p^{0}}{d \tau} \frac{d p^{0}}{d \tau}+\sum_{i=1}^{3} g_{i i} \frac{d p^{i}}{d \tau} \frac{d p^{i}}{d \tau}\right) \\
P=-\frac{e^{2}}{6 \pi m^{2}}\left[\left(\frac{d p^{0}}{d \tau}\right)^{2}-\frac{d \vec{p}}{d \tau} \frac{d \vec{p}}{d \tau}\right] \\
P=-\frac{e^{2}}{6 \pi m^{2}}\left[\left(\frac{d p^{0}}{d \tau}\right)^{2}-\left|\frac{d \vec{p}}{d \tau}\right|^{2}\right] \\
P=-\frac{e^{2}}{6 \pi m^{2}}\left[\left(\frac{d E}{d \tau}\right)^{2}-\left|\frac{d \vec{p}}{d \tau}\right|^{2}\right]
\end{gathered}
$$

obtemos

$$
P=-\frac{e^{2}}{6 \pi m^{2}} \gamma^{2}\left[\left(\frac{d E}{d t}\right)^{2}-\left|\frac{d \vec{p}}{d t}\right|^{2}\right] .
$$

Sabendo que

$$
\frac{d E}{d t}=\frac{\vec{p}}{E} \frac{d|\vec{p}|}{d t}
$$

e substituindo essa última expressão na equação 133, alcançamos

$$
P=\frac{e^{2}}{6 \pi m^{2}} \gamma^{2}\left[\left|\frac{d \vec{p}}{d t}\right|^{2}-|\vec{v}|^{2}\left(\frac{d \vec{p}}{d t}\right)^{2}\right]
$$

que representa a expressão relativística para a potência total irradiada pela partícula. Em geral, essa equação é aplicada para os aceleradores de partículas carregadas 36 .

Partindo da equação (132), podemos ainda alcançar outro resultado relevante. Nesse caso, fazemos inicialmente 


$$
\begin{array}{r}
\frac{d \gamma}{d \tau}=\gamma \frac{d \gamma}{d t}=-\frac{1}{2} \gamma\left(1-|\vec{v}|^{2}\right)^{-3 / 2}(-2|\vec{v}|) \frac{d|\vec{v}|}{d t} \\
\frac{d \gamma}{d \tau}=|\vec{v}| \gamma\left[\left(1-|\vec{v}|^{2}\right)^{-1 / 2}\right]^{3} \frac{d[(\vec{v} \cdot \vec{v})]^{1 / 2}}{d t} \\
\frac{d \gamma}{d \tau}=|\vec{v}| \gamma^{4} \frac{1}{2}(\vec{v} \cdot \vec{v})^{-1 / 2}\left[\frac{d \vec{v}}{d t} \cdot \vec{v}+\vec{v} \cdot \frac{d \vec{v}}{d t}\right]
\end{array}
$$

e obtemos:

$$
\frac{d \gamma}{d \tau}=\gamma^{4}(\vec{v} \cdot \vec{a})
$$

Os próximos passos são destinados à obtenção dos termos $\left|\frac{d \vec{p}}{d \tau}\right|^{2}$ e $\left(\frac{d E}{d \tau}\right)^{2}$. Para alcançarmos uma expressão de $\left|\frac{d \vec{p}}{d \tau}\right|^{2}$ fazemos

$$
\begin{gathered}
\left|\frac{d \vec{p}}{d \tau}\right|^{2}=\left|m \frac{d(\gamma \vec{v})}{d \tau}\right|^{2}=m^{2}\left|\vec{v} \frac{d \gamma}{d \tau}+\gamma \frac{d \vec{v}}{d \tau}\right|^{2} \\
\left|\frac{d \vec{p}}{d \tau}\right|^{2}=m^{2}\left|\vec{v} \gamma^{4}(\vec{v} \cdot \vec{a})+\gamma^{2} \vec{a}\right|^{2}
\end{gathered}
$$

o que resulta na expressão:

$$
\left|\frac{d \vec{p}}{d \tau}\right|^{2}=m^{2}\left[|\vec{v}|^{2} \gamma^{8}(\vec{v} \cdot \vec{a})^{2}+2 \gamma^{6}(\vec{v} \cdot \vec{a})^{2}+\gamma^{4}|\vec{a}|^{2}\right] .
$$

(137)

A expressão de $\left(\frac{d E}{d \tau}\right)^{2}$, por sua vez, pode ser conseguida por meio dos seguintes passos:

$$
\begin{gathered}
\left(\frac{d E}{d \tau}\right)^{2}=\left[\frac{d(\gamma m)}{d \tau}\right]^{2}=\left[\frac{d(\gamma m)}{d \tau}\right]^{2}=\left[m \gamma^{4}(\vec{v} \cdot \vec{a})\right]^{2} \\
\left(\frac{d E}{d \tau}\right)^{2}=m^{2} \gamma^{8}(\vec{v} \cdot \vec{a})^{2}
\end{gathered}
$$

Substituindo as expressões 137 e 138 na equação (132) como se segue

$$
\begin{gathered}
P=\frac{e^{2}}{6 \pi m^{2}}\left\{m^{2}\left[\left(|\vec{v}|^{2}-1\right) \gamma^{8}(\vec{v} \cdot \vec{a})^{2}+2 \gamma^{6}(\vec{v} \cdot \vec{a})^{2}+\gamma^{4}|\vec{a}|^{2}\right]\right\} \\
P=\frac{e^{2}}{6 \pi}\left[\gamma^{6}(\vec{v} \cdot \vec{a})^{2}+\gamma^{4}|\vec{a}|^{2}\right]=\frac{e^{2}}{6 \pi} \gamma^{6}\left[(\vec{v} \cdot \vec{a})^{2}+\gamma^{-2}|\vec{a}|^{2}\right] \\
P=\frac{e^{2}}{6 \pi} \gamma^{6}\left[|\vec{a}|^{2}-|\vec{v}|^{2} \cdot|\vec{a}|^{2}+(\vec{v} \cdot \vec{a})^{2}\right]
\end{gathered}
$$

e empregando a identidade de Lagrange, na qual

$$
|\vec{v} \times \vec{a}|^{2}+|\vec{v} \cdot \vec{a}|^{2}=|\vec{v}|^{2}|\vec{a}|^{2}
$$

alcançamos:

$$
P=\frac{e^{2}}{6 \pi} \gamma^{6}\left[|\vec{a}|^{2}-|\vec{v} \times \vec{a}|^{2}\right] .
$$

A expressão 139 foi obtida em 1898 por Alfred-Marie Liénard e hoje é conhecida como a fórmula ou generalização de Liénard.

\section{Comparação com a potência emitida por uma fonte escalar interagindo com campo escalar não-massivo de Klein-Gordon}

Empregando boa parte dos passos desenvolvidos nas seções anteriores, podemos estudar outras interações entre matéria e campo, como é o caso da interação de uma fonte escalar pontual interagindo com o campo não-massivo de Klein-Gordon 4 . Esse campo, fundamentado na interpretação de Feynman-Stueckelberg, tem importante papel na descrição do comportamento de partículas de spins nulos, como os mésons $\left(\pi^{-}, \pi^{+}\right.$e $\left.\pi^{0}\right)$ e, por consequência, em abordar determinados campos bosônicos 5 .

No caso da interação em questão, consideremos um campo escalar sem massa $\phi(x)$ interagindo com uma fonte puntual acelerada $J(x)$. Em foco, a densidade de lagrangeana que descreve esse sistema é dada por:

$$
£=\frac{1}{2} \partial^{\mu} \phi \partial_{\mu} \phi+\frac{1}{2} J(x) \phi(x) .
$$

Fazendo uso das equações de Euler-Lagrange, podemos alcançar a expressão

$$
\partial^{\mu} \partial_{\mu} \phi=J
$$

conhecida como equação de Klein-Gordon não homogênea para o campo escalar clássico não-massivo gerado por uma fonte escalar $J(x)$.

Aplicando o formalismo das funções de Green, a expressão para o campo escalar não-massivo será:

$$
\phi(x)=\frac{q}{4 \pi U_{\mu}\left(\tau_{0}\right)\left(x-z\left(\tau_{0}\right)\right)^{\mu}} .
$$

Nessa última expressão 142, o termo $\tau_{0}$ representa o tempo próprio da fonte no instante da emissão da radiação, evento este associado à posição $z^{\mu}\left(\tau_{0}\right)$ da carga no espaço-tempo de Minkowski, enquanto $U_{\mu}$ refere-se à quadrivelocidade. Para a obtenção do vetor de Poynting, o tensor energia-momento canônico é dado por [35]:

$$
T_{\mu \nu}=\frac{\partial £}{\partial\left(\partial^{\mu} \phi\right)} \partial_{\nu} \phi-\eta_{\mu \nu} £ .
$$

Empregando alguns dos passos apresentados para obtenção da potência de Larmor, alcançaremos

$$
P=\oiint \vec{S} d \vec{A}=\frac{q^{2} a^{2}}{12 \pi}
$$

\footnotetext{
${ }^{4}$ Campo descrito pela equação de Klein-Gordon proposta originalmente em 1926 pelo físico sueco Oskar Klein (1894-1977) e pelo físico alemão Walter Gordon (1893-1939) para descrever elétrons relativísticos. Convém mencionar que algumas vezes, essa equação é também conhecida como equação de Klein-Gordon-Fock devido ao físico soviético Vladimir Fock (1898-1974) que também obteve a mesma expressão.

5 Menciona-se que embora o campo de Klein-Gordon não tenha análogo clássico por ser estritamente quântico, o mesmo pode ser tratado como campo clássico 37 .
} 


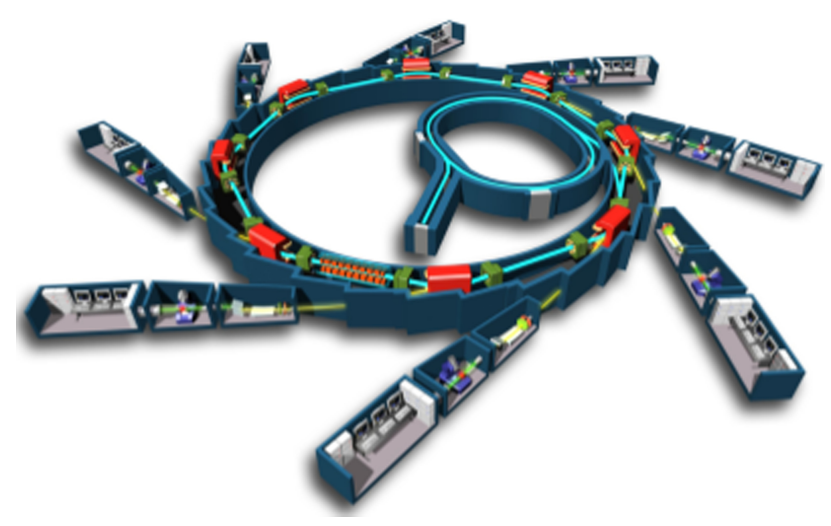

Figura 10: Esquema estrutural do acelerador de elétrons Synchrotron SOLEIL na França [39].

Observe que o resultado 144 é igual a metade do valor clássico da potência irradiada para o campo eletromagnético $A^{\mu}$ 129. Na verdade, isso já era um resultado esperado, haja vista que o campo eletromagnético enquanto campo vetorial não-massivo, possui dois graus de liberdade associados aos dois graus de polarização do mesmo 38.

\section{Radiação Síncrotron}

Há diversas aplicações dos fenômenos da radiação no mundo contemporâneo que reconhecidamente mereceriam um texto exclusivo para abordá-las tanto no que se refere às atuais tecnologias quanto às investigações científicas que as utilizam. De todo modo, discutimos brevemente nessa seção sobre a radiação síncrotron em razão do imponente projeto Sirius.

Em termos gerais, a radiação síncrotron é aquela emitida por uma carga movendo-se com velocidade relativística ao longo de uma trajetória curva. Particularmente, essa condição é aplicável para partículas circulando em aceleradores de elétrons que, por sua vez, apresentam raio de curvatura da ordem de dezenas de metros.

Para se obter essa radiação, os elétrons a serem acelerados são gerados pelo aquecimento de uma liga metálica e, em seguida, são conduzidos para o anel de aceleração, no qual os elétrons têm suas velocidades elevadas antes de serem enviadas para o anel principal (figura 10. Nessa estrutura principal, as partículas passam a viajar com velocidades relativísticas por um sistema de tubos de vácuo sendo devidamente guiadas por uma rede magnética; quando suas trajetórias são desviadas, os elétrons emitem radiações que são coletadas pelas linhas de luz (localizadas em estações fora do anel principal). A obtenção do feixe de radiação com a frequência de interesse é alcançada condicionando a radiação emitida a um complexo sistema de espelhos, fendas e monocromadores.

Desde 1997, a ciência brasileira já tem acesso à tecnologia da radiação síncrotron, com a inauguração do
Laboratório Nacional de Luz Síncrotron (LNLS) $\sqrt{6}$ situado na cidade de Campinas, onde o acelerador UVX (sigla de "Ultravioleta do Raio-x") opera. Está previsto para 2018 a inauguração do Sirius (referência à estrela mais brilhante no céu noturno, localizada na constelação de Canis Major), uma superfonte moderna de luz síncrotron que representará o projeto científico mais ambicioso do país até hoje e que permitirá à comunidade científica, tecnológica e industrial do país colocar-se na fronteira da ciência, da tecnologia e da inovação em nível global 40]

As principais aplicações de luz síncrotron estão nas áreas de física da matéria condensada, ciência dos materiais, química, farmácia, paleontologia, nanotecnologia, biologia (molecular e estrutural), agricultura (sementes e fibras), energia (análise de rochas da camada pré-sal e desenvolvimento de baterias) e medicina (em especial, na obtenção de imagens detalhadas de tecidos).

Convém mencionar que a radiação síncrotron também pode ter origem natural, sendo produzida, por exemplo, por objetos astronômicos como quasares, núcleos de galáxias ativas e remanescentes de supernovas (pulsares) [41].

\section{Considerações finais}

Desenvolveu-se no presente texto, os passos necessários para abordar o fenômeno da emissão de radiação devido a uma carga elétrica acelerada no espaço-tempo de Minkowski; em foco, obtemos mediante a teoria clássica de campos a potência total irradiada num referencial instantaneamente em repouso com a carga (potência de Larmor) além da generalização de Liénard.

Os aspectos físicos gerais dessa interação são relevantes por permitirem compreender inúmeros fenômenos clássicos envolvendo radiação (térmica, sincrotrônica, etc.)

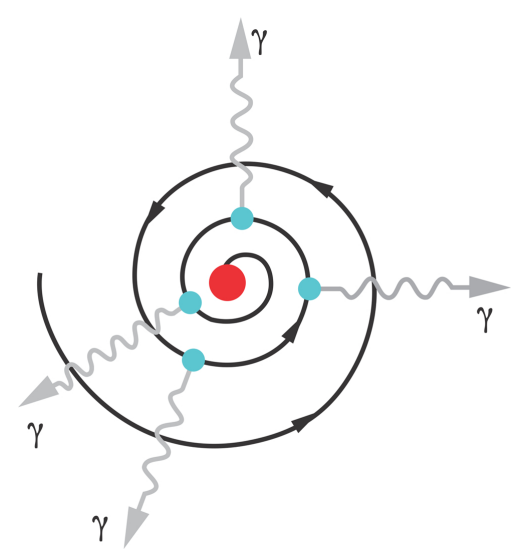

Figura 11: Inconsistência do modelo atômico de Rutherford, no qual, pela eletrodinâmica clássica, o elétron por ter aceleração não nula, irradia energia e descreve uma trajetória espiral em direção ao núcleo até colapsar com o mesmo [43].

\footnotetext{
6 O LNLS é administrado pelo Centro Nacional de Pesquisa em Energia e Materiais (CNPEM), uma organização social supervisionada pelo Ministério da Ciência, Tecnologia, Inovações e Comunicações (MCTIC).
} 
e espalhamento (Rayleig, Thomson, ressonância, etc.). Ademais, a conclusão de que cargas pontuais aceleradas irradiam e, por consequência, perdem energia, teve um importante papel no desenvolvimento de um modelo atômico fisicamente consistente. Por exemplo, os modelos atômicos do tipo planetário como aquele proposto por Ernest Rutherford (1871-1937) não conseguiam justificar a estabilidade da matéria, pois se os elétrons orbitassem um núcleo, seriam constantemente acelerados e, por conseguinte, perderiam energia pela emissão de radiação eletromagnética, de tal forma que os raios de suas órbitas diminuiriam e a frequência da radiação aumentaria até que essas partículas fossem capturadas pelo núcleo resultando assim no colapso do átomo (figura 11). Para uma órbita de ordem atômica, esse colapso ocorreria em um tempo inferior a 1 nanossegundo 42]. O problema da estabilidade do átomo só veio a ser esclarecido com o advento da Mecânica Quântica, através dos modelos atômicos de Niels Bohr (1885-1962) e Erwin Schrödinger (1887-1961).

Por fim, ainda que a abordagem empregada tenha objetivado, essencialmente, o estudo do fenômeno da radiação de carga acelerada por meio da Teoria Clássica de Campos, o desenvolvimento apresentado pode ser proficuamente utilizado como um passo inicial para investigar diferentes campos e suas interações com a matéria, assim como um texto didático preparatório para o estudo da Teoria Quântica de Campos.

\section{Agradecimentos}

Enfocamos nossos agradecimentos à produtiva parceria entre o Instituto Federal do Pará (Campus Santarém) e o Instituto de Engenharia e Geociências da Universidade Federal do Oeste do Pará (em especial, o Laboratório de Sinais e Sistemas - LABSIS).

Ademais, agradecemos o ilustre Professor Luís Carlos Bassalo Crispino pelas profícuas discussões relativas ao tema.

\section{Referências}

[1] A.S.T. Pires, Evolução das Ideias da Física (Livraria da Física, São Paulo, 2011).

[2] J.C. Maxwell, Transactions of the Cambridge Philosophical Society 10 (1856). Republicado in: W.D. Niven (editor) The Scientific Papers of James Clerk Maxwell (Cambridge University Press, Cambridge, 1890; reprint, 2010), v. 1 , p. $155-229$.

[3] J.C. Maxwell, Philosophical Magazine 21 (1861). Republicado in: W.D. Niven (editor) The Scientific Papers of James Clerk Maxwell (Cambridge University Press, Cambridge, 1890; reprint, 2010), v. 1, p. 451-490.

[4] J.C. Maxwell, Royal Society Transactions 45 (1864). Republicado in: W.D. Niven (editor) The Scientific Papers of James Clerk Maxwell (Cambridge University Press, Cambridge, 1890; reprint, 2010), v. 1, p. 526-597.
[5] J.C. Maxwell, A Treatise on Electricity \& Magnetism (Clarendon, Oxford, 1873); reprint da terceira edição (1891) (Dover, New York, 1954).

[6] V.A. Bezerra, Scientiae Studia 4, 2 (2006).

[7] B. Riemann, Philosophical Magazine 34, 368 (1867).

[8] L. Lorenz, Philosophical Magazine 34, 287 (1867).

[9] L. Lorenz, Journal Fur Die Reine Und Angewandte Mathematik 58, 329 (1861).

[10] E. Wiechert, Archives Neerlandaises Sci Exactes et Naturelles 5, 549 (1900).

[11] J. Larmor, Philosophical Magazine 44, 5 (1897).

[12] F. Caruso and V. Oguri, Física Moderna: Origens Clássicas e Fundamentos Quânticos (Elsevier, Rio de Janeiro, 2006).

[13] A. Liénard, L'Éclairage Électrique 16, 5, 53, 106 (1898).

[14] J.M.F. Bassalo, Crônicas da Física: Tomo I (Editora da Universidade Federal do Pará, Belém, 1987).

[15] J.M.F. Bassalo, Eletrodinâmica Clássica (Editora Livraria da Física, 2007).

[16] M. Born, Annalen der Physik, 30, 1 (1909).

[17] W. Pauli, Enzyklopädie der Mathematischen Wissenscahften, Volume 5 (Teubner, Leipzig, 1918).

[18] M.V. Laue, Relativitätstheorie (Vieweg, Braunschweig, 1919).

[19] J.C. Rodrígues, Princípio de Equivalência, Partículas Não Massivas com $E=0$ e Partículas Massivas com $E<m c^{2}$ em Espaços Curvos e Aplicações. Tese de Doutorado, Instituto de Física Teórica, Universidade Estadual Paulista, 2002.

[20] H. Bondi and T. Goldi, Proceedings of the Royal Society A229, 416 (1955).

[21] T. Fulton and F. Rohrlich, Annals of Physics 9, 499 (1960).

[22] F. Rohrlich, Nuovo Cimento 21, 811 (1961).

[23] F. Rohrlich, Annals of Physics 22, 169 (1963).

[24] F.B. Silsbee, Journal of Research of the National Bureau of Standards - Engineering and Instrumentation, 66C, 2 (1962).

[25] R.G. Littlejohn, Gaussian, SI and Other Systems of Units in Electromagnetic Theory, available in http://bohr .physics.berkeley.edu/classes/221/ 1112/notes/emunits.pdf, acess in september 28, 2017.

[26] R.D. Inverno, Introducing Einstein's Relativity (Clarendon Press, Oxford, 1992).

[27] W. Greiner and J. Reinhardt, Field Quantization (Springer, Heidelberg, 1996).

[28] H. Goldstein, C. Poole and J. Safko, Classical Mechanics (Addison-Wesley, New York, 2001).

[29] J.D. Jackson, Classical Electrodynamics (John Wiley \& Sons, Inc., New York, 1998).

[30] N.A. Lemos, Mecânica Analítica (Livraria da Física, São Paulo, 2007).

[31] R.V. Churchill, Complex Variables and Applications (McGraw-Hill, New York, 2014).

[32] A.O. Barut, Electrodynamics and Classical Theory of Fields and Particles (Dover Publications, Inc., New York, 1964).

[33] K.D. Machado, Teoria do Eletromagnetismo. Vol 3 (UEPG, Ponta Grossa, 2000).

[34] D.P. Meira Filho, Cálculo da Potência Irradiada por Correntes Clássicas Uniformemente Acelerados no EspaçoTempo de Minkowski Interagindo com Campos Bosônicos 
Sem Massa. Trabalho de Conclusão de Curso, Faculdade de Física, Instituto de Ciências Exatas e Naturais, Universidade Federal do Pará, 2003.

[35] L.C. Bassalo Crispino, Quantização a Baixas Frequências de Campos Bosônicos no Espaço-Tempo de Schwarzschild e Aplicações. Tese de Doutorado, Instituto de Física Teórica, Universidade Estadual Paulista, 2001.

[36] J. Frenkel, Princípios de Eletrodinâmica Clássica (Edusp, São Paulo, 1996).

[37] L.H. Ryder, Quantum Field Theory (Cambridge University Press, Cambridge, 1985).

[38] L. Landau e E. Lifchitz, Curso de Física: Teoria do Campo (Mir, Moscou, 1980).

[39] Synchrotron Soleil, available in https://www. synchrotron-soleil.fr/, access in october 21, 2017.

[40] Centro Nacional de Pesquisa em Energia e Materiais, Sirius: A Superfonte de Luz Síncrotron no Brasil, Disponível em http://cnpem.br/sirius-superfonte-luzsincrotron-brasil/, acesso em 20/10/2017.

[41] G.S.B. Kogan, in: Synchrotron Radiation Theory and Its Development, edited by V. A. Bordovitsyn (Singapura, World Scientific, 1999) p. 385-386.

[42] H.M. Nussenzveig, Curso de Física Básica: Ótica, Relatividade e Física Quântica (Edgard Blucher, São Paulo, 2002).

[43] P.A. Tipler e R.A. Llewellyn, Física Moderna (LTC, Rio de Janeiro, 2012). 